\title{
Coming Together: RNAs and Proteins Assemble under the Single-Molecule Fluorescence Microscope
}

\author{
Ameya P. Jalihal, ${ }^{1,2}$ Paul E. Lund, ${ }^{2}$ and Nils G. Walter ${ }^{2,3}$ \\ ${ }^{1}$ Cellular and Molecular Biology Graduate Program, University of Michigan, Ann Arbor, Michigan 48109 \\ ${ }^{2}$ Single Molecule Analysis Group, Department of Chemistry, University of Michigan, Ann Arbor, Michigan 48109 \\ ${ }^{3}$ Center for RNA Biomedicine, University of Michigan, Ann Arbor, Michigan 48109 \\ Correspondence: nwalter@umich.edu
}

\section{SUMMARY}

RNAs, across their numerous classes, often work in concert with proteins in RNA-protein complexes (RNPs) to execute critical cellular functions. Ensemble-averaging methods have been instrumental in revealing many important aspects of these RNA-protein interactions, yet are insufficiently sensitive to much of the dynamics at the heart of RNP function. Singlemolecule fluorescence microscopy (SMFM) offers complementary, versatile tools to probe RNP conformational and compositional changes in detail. In this review, we first outline the basic principles of SMFM as applied to RNPs, describing key considerations for labeling, imaging, and quantitative analysis. We then sample applications of in vitro and in vivo single-molecule visualization using the case studies of pre-messenger RNA (mRNA) splicing and RNA silencing, respectively. After discussing specific insights single-molecule fluorescence methods have yielded, we briefly review recent developments in the field and highlight areas of anticipated growth.

\section{Outline}

1 Introduction

2 General considerations for applying singlemolecule fluorescence microscopy to RNA-protein interactions

3 Application example of in vitro singlemolecule fluorescence: The spliceosome

4 Deeper considerations for studying single RNPs in vitro
5 Single-RNP visualization in living cells

6 Application example of in vivo singlemolecule fluorescence: RNA silencing

7 Conclusions and outlook

References 
A.P. Jalihal et al.

\section{INTRODUCTION}

Contemplating the original "central dogma" of molecular biology (Crick 1970), one could easily come away with the impression that RNA serves only as a genetic messenger, conveying the information stored in DNA by helping convert it into functional protein actuators. Over the past few decades, RNA has, however, turned out to be much more than a mere genetic messenger, with the vast majority of RNA transcripts from eukaryotic genomes having no protein coding capacity. The many classes of such noncoding RNAs-including ribosomal RNA (rRNA), transfer RNA (tRNA), short noncoding RNA, such as small RNA (sRNA), microRNA (miRNA), and long noncoding RNA (lncRNA), to name a few-perform a multitude of catalytic, regulatory, and architectural functions. To help carry out their func- tions, each of these classes of RNA participates in a variety of interactions with one or more protein partners. As an example, the messenger RNAs (mRNAs) that inspired the central dogma are themselves dependent on interactions with a multitude of proteins throughout their life cycle, from biogenesis, editing, and regulation to decay. Given how intimately the biological activity of a given RNA is connected to interactions with these protein partners, discerning the nature of these interactions is vital to understanding the biology of RNA-dependent cellular pathways.

Many long-standing techniques for studying ribonucleoproteins (RNPs), such as electrophoretic gel shift assays or ultraviolet (UV) cross-linking, are most suitable for interactions that are tight and uniform in binding mode and composition, which is not the case for many complex biological processes. In fact, it is becoming increasingly clear
A

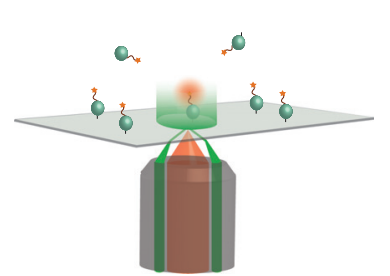

Objective-type TIRFM

B

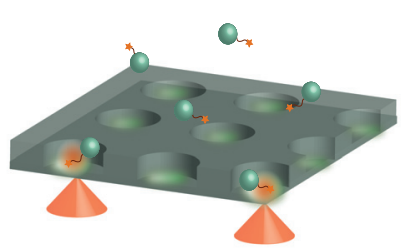

Zero-mode waveguides

D

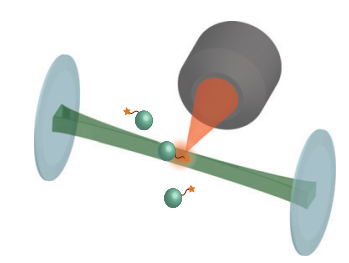

Light-sheet illumination

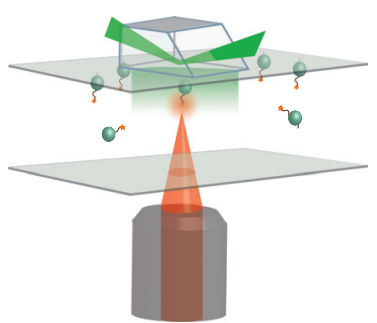

Prism-type TIRFM

C

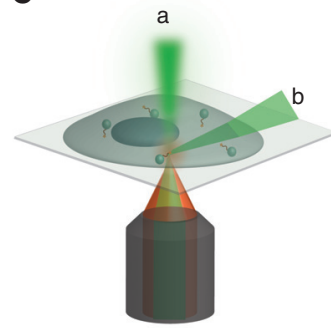

a, Epifluorescence. b, HILO

E

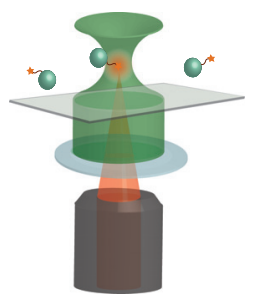

Confocal illumination
$\mathbf{F}$

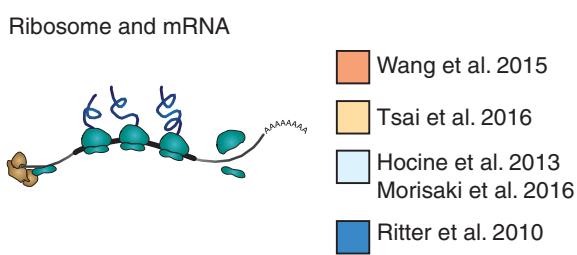

RNA-induced silencing complex

Salomon et al. 2015

Lu and Tsourkas 2009 Pitchiaya et al. 2012, 2017
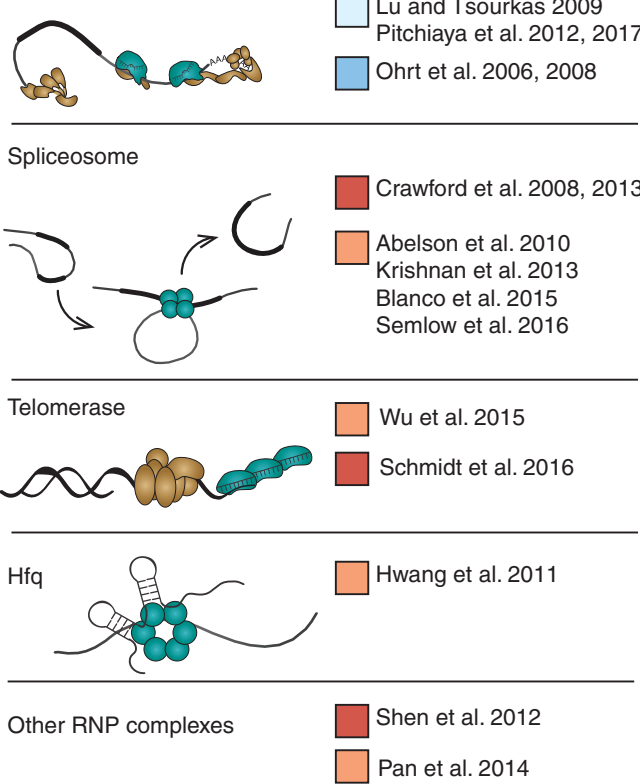

Figure 1. Overview of single-molecule microscopies applied to study ribonucleoproteins (RNPs). (A) Objective- and prism-type total internal reflection fluorescence microscopy (TIRFM) configurations. $(B)$ Zero-mode waveguides. $(C)$ a, Epifluorescence; b, highly inclined and laminated optical sheet (HILO) illumination. $(D)$ Light-sheet illumination. (E) Confocal illumination. $(F)$ Selected studies on RNPs that have used fluorescence-based, single-molecule methods. Colored boxes indicate the type of microscopy configuration used. 
that the dynamic and transient nature of competing interactions between biomolecules is at the heart of cellular pathways, such as pre-mRNA splicing (Pan et al. 2014; Semlow et al.2016), mRNA translation (Agirrezabala and Valle 2015; Tsai et al. 2016), RNA chaperoning (Shen et al. 2012), and RNA silencing and regulation (Hwang et al. 2011; Jens and Rajewsky 2015). Particularly as compositional and structural snapshots of large, multicomponent biomolecular machines such as the spliceosome come into focus thanks to new structural data, the need for a deeper understanding of the compositional and conformational dynamics of RNPs becomes increasingly apparent. Two decades of advances in single-molecule fluorescence microscopy (SMFM) have now given us the tools to address this need.

\section{GENERAL CONSIDERATIONS FOR APPLYING SINGLE-MOLECULE FLUORESCENCE MICROSCOPY TO RNA-PROTEIN INTERACTIONS}

SMFM techniques are well-suited to study molecular processes that occur in multiple steps, proceed via parallel reaction pathways, show transient excursions to distinct states, and/or contain varying components, all of which are frequently true of RNA-protein interactions (Wahl et al. 2009; Moffitt et al. 2010). The most important advantage is that these methods allow heterogeneous molecular "behaviors" to be uncovered in asynchronous populations of molecules proceeding through multiple reaction pathways at different rates (Lu et al. 1998; Larson et al. 2014). These single-molecule behaviors can be scrutinized at a spatiotemporal resolution inaccessible to bulk techniques, leading to insights that can be directly compared with bulk data by postacquisition time and ensemble averaging.

The general benefits and specifics of performing singlemolecule studies are beyond the scope of this review, but have been reviewed elsewhere (Roy et al. 2008; Walter et al. 2008; Liu et al. 2015). We instead will focus on the salient features of SMFM and practical considerations for its application to the study of RNPs.

A variety of SMFM approaches exist today, the exact choice of which depends largely on the study design and experimental goals. SMFM has been used to investigate numerous RNPs, including those involved in transcription, splicing, RNA modification and editing, chaperone activity, and RNA interference (Fig. 1). Despite obvious differences in the underlying biology, key experimental elements common to all of these studies include illumination, a strategy for labeling the biomolecule(s) of interest, detection of fluorescence, and data analysis, as discussed below.

\subsection{Illumination and Detection}

By design, SMFM approaches aim to characterize the behavior of individual molecules. However, detecting the signal from an individual molecule in the background of other molecules, potential contaminants, and instrumental noise can be challenging. Fluorescence itself facilitates distinguishing genuine signal from background as light emitted from fluorescently tagged biomolecules is red-shifted relative to the wavelength of laser light used for excitation. This spectral separation (i.e., "Stokes shift") between the excitation and emission wavelength maxima makes genuine fluorescence signals easily distinguishable from background scattering of the sample medium.

In addition to spectral isolation of the relevant signal, limiting the volume of illumination improves signal-tonoise by decreasing the contribution of out-of-focus emission. Of the illumination modalities that achieve selective volume illumination, total internal reflection fluorescence microscopy (TIRFM) is perhaps the most widely implemented (Fig. 1A). In TIRFM, the excitation beam is totally internally reflected near the sample plane (Toomre 2012). An evanescent field of excitation light is generated at the surface that extends $\sim 100 \mathrm{~nm}$ into the sample. This evanescent field illuminates molecules immobilized at the imaging surface, thus minimizing background fluorescence from molecules deeper in solution. The level of background fluorescence observed in TIRFM experiments is dependent on the concentration of excess fluorescently labeled biomolecules in solution and provides good signal-to-noise up to fluorophore concentrations of $\sim 100 \mathrm{~nm}$. For RNA and proteins that interact with high affinity, this effective concentration limit does not pose a problem. However, physiologically relevant interactions often involve micromolar affinities of binding partner(s). Zero-mode waveguide (ZMW) technology allows single-molecule resolution even in solutions with high concentrations of fluorescent components (Levene et al. 2003), and have been particularly useful in studies of the ribosome (Fig. 1B) (Tsai et al. 2016).

Another strategy to limit out-of-plane fluorescence by controlling the illumination area is highly inclined and laminated optical sheet (HILO) illumination (Fig. 1C) (Tokunaga et al. 2008). In HILO microscopy, the illuminated region is thicker than it is for TIRFM (e.g., $\sim 7 \mu \mathrm{m}$ for an illuminated area with a diameter of $20 \mu \mathrm{m}$ ), and contains a $z$-component, which allows for imaging of molecules that lie micrometers above the coverslip surface. These characteristics make HILO microscopy particularly suited for studying samples with three-dimensional spatial distributions, such as single molecules inside live cells. Even more sample penetration depth can be achieved by light-sheet 
fluorescence microscopy (LSFM), in which a focused sheet of light is used to illuminate only a thin section of a thick sample for single-molecule detection (Fig. 1D) (Ritter et al. 2010). Confocal microscopy uses pinholes to limit the volume in which single molecules are detected (Fig. 1E). In addition to two-dimensional (2D) scanning, confocal microscopy is typically used in the context of fluorescence correlation spectroscopy (FCS) and two-color fluorescence cross-correlation spectroscopy (FCCS), in which a highsensitivity, photon-counting point detector generates time-resolved intensity measurements from the entire illuminated confocal volume (Liu et al. 2015; Gonzalez Bardeci et al. 2017).

\subsection{Labeling Biomolecules of Interest}

Another key requirement of SMFM is that one or more of the biological components in the system being studied must be fluorescently labeled.

The ever-increasing number of small-molecule fluorescent dyes available provide flexibility and choice for labeling. CyDye, Alexa Fluor, DyLight, and ATTO are perhaps the most popular in the single-molecule fluorescence field because of their brightness, photostability, and the commercial availability of derivatives for bioconjugation. Their $\mathrm{pH}$ tolerance also makes them suitable for fluorescent labeling in vivo (see Sec. 5). Additionally, multiple fluorophore pairs (e.g., Cy3 and Cy5; Dy547 and Cy5) have spectral properties that permit their use as single-molecule Förster resonance energy transfer (smFRET) reporters, allowing for sensitive monitoring of nanometer-scale changes in distance (Lakowicz 2006; McCann et al. 2010). Experiments using such small-molecule dyes typically use one of several oxygen-scavenging systems (OSSs) to improve dye photophysical performance and longevity (Rasnik et al. 2006; Aitken et al. 2008). However, the choice of an OSS must also keep the biological system in mind, including the potential effect of $\mathrm{pH}$ changes or sensitivity to nuclease contamination (Swoboda et al. 2012; Senavirathne et al. 2015). For example, a very common OSS based on glucose oxidase is inappropriate for experiments with whole-cell extracts (WCEs) because the activity of endogenous kinases quickly phosphorylates the glucose, depleting ATP from solution and thus inhibiting processes such as splicing (Crawford et al. 2008; Abelson et al. 2010).

It should be noted that in the context of RNP studies, although the most obvious choice may be to label the RNA and protein components that are directly interacting in multicomponent systems, it can be advantageous to label, for example, a strongly associated component whose presence or absence is then used to distinguish complexes of interest from other closely related species. In the following, we provide a brief overview of commonly used fluorescentlabeling strategies and provide particular considerations for the study of RNPs.

\subsubsection{Labeling RNA Components}

Labeling sites must be chosen so as to avoid influencing interactions between the RNA and its binding partner(s), or perturbing secondary or tertiary interactions within the RNA or its conformational dynamics (Al-Hashimi and Walter 2008; Mustoe et al. 2014; Darre et al. 2016). For example, $2^{\prime}$ modifications on the ribose sugar can alter the thermal stability of double-stranded regions (Hendrix et al. 1995; Fauster et al. 2012), whereas attachment of fluorophores at $5^{\prime}$ or $3^{\prime}$ termini can increase duplex stability through stacking of the fluorophores on the terminal base-pairs (Cisse et al. 2012; Liu and Lilley 2017). Such alterations to thermal stability can potentially have functional consequences. In the context of miRNA guide strand selection and loading into the RNA-induced silencing complex (RISC), for example, a $5^{\prime}$ terminal fluorophore disfavored use of the labeled strand as a guide (Custer and Walter 2017). Further, in contrast to DNA where canonical Watson-Crick base-pairing dominates, RNA secondary and tertiary structures show an astonishing variety of noncanonical base-pairing interactions (Leontis et al. 2002), including interactions with the sugar edge (Nissen et al. 2001). As such, it is useful to examine all available structural data when deciding which point of attachment (e.g., $2^{\prime} \mathrm{OH}$, phosphate, or pyrimidine $\mathrm{C} 5$; and position in the sequence) is likely to result in the least perturbation.

Chemical synthesis of oligonucleotides provides a direct route for preparing site-specifically labeled RNA. Fluorophore-labeled nucleotides can be directly incorporated during synthesis, or fluorophores can be conjugated after synthesis through the use of reactive groups present on modified nucleotides (Fig. 2A) (Paredes et al. 2011; Edwards and Sigurdsson 2014). Reactive groups such as primary amines and azides and common conjugation chemistries such as $N$-hydroxysuccinimide esters and "click" chemistry allow fluorophores to be attached after RNA synthesis (Fig. 2A,B) (Kolb et al. 2001; Rinaldi et al. 2015). Alternatively, a limited number of generalized labeling chemistries that use natural features of the RNA, such as the $5^{\prime}$ phosphate, naturally occurring 4-thiouridine in some tRNAs, and the $3^{\prime}$ vicinal diol, can be used for labeling (Fei et al. 2010; Hermanson 2013; Willkomm and Hartmann 2014). A significant limitation, however, is that chemical synthesis is only a viable option for relatively short RNAs $(<80 \mathrm{nt})$. As a result, longer RNAs used for in vitro studies are frequently prepared by enzymatic, splint-mediated ligation, either from multiple chemically synthesized oligonucleotides 
A<smiles></smiles>

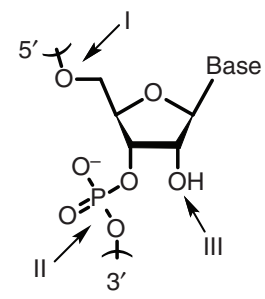<smiles>Cn1c(N)nc2c(N)ncnc21</smiles><smiles>CCn1cc(C(=O)O)c(=O)[nH]c1=O</smiles>

Modifications:

I. \& II. $\mathrm{NNH}_{2},-\mathrm{C} \equiv \mathrm{CH},-\mathrm{N}_{3},-\mathrm{SH}$; biotin-TEG, digoxigenin; fluorophore III. $-\mathrm{NH}_{2},-\mathrm{N}_{3}$ IV. $-\mathrm{NH}_{2} \sim \sim \mathrm{NH}_{2},-\mathrm{N}_{3}$ V. $\sim \sim \mathrm{NH}_{2}, \longrightarrow \mathrm{NH}_{2}, \sim \mathrm{N}_{3},=\backsim \mathrm{C} \equiv \mathrm{CH}$, strained alkyne VI. S

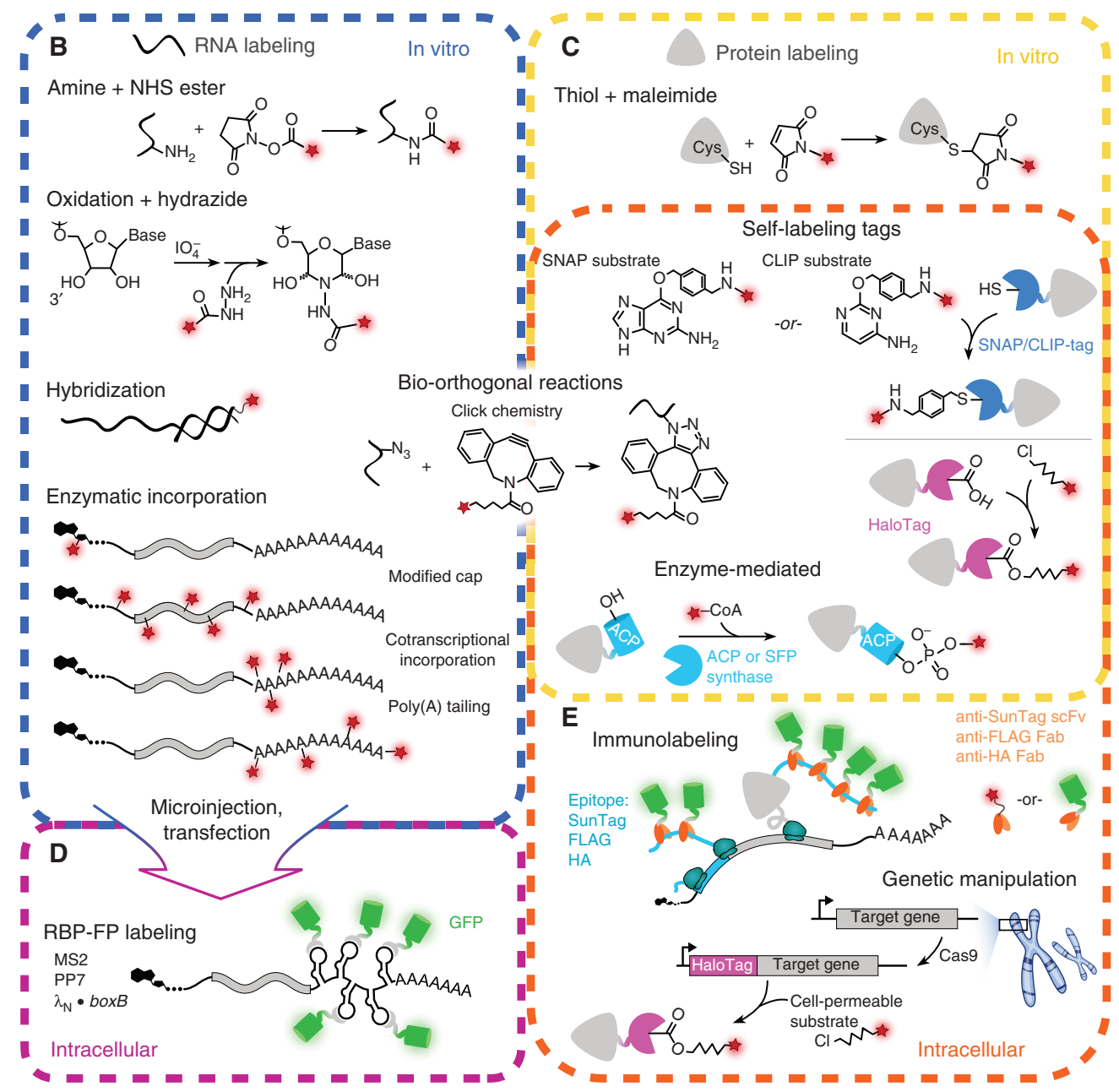

Figure 2. Strategies for labeling RNA and protein components for single-molecule fluorescence. (A) Modifications are possible at various positions on RNA molecules prepared through chemical synthesis, including the $5^{\prime}$ and $3^{\prime}$ termini (I and II, respectively), the $2^{\prime}$ position of the sugar (III), and various positions on the nucleobase (IV, V, and VI), among others. Modifications can include reactive chemical moieties that are used for subsequent conjugation reactions, small molecules like biotin and digoxigenin for immobilization, or direct attachment of fluorophore dyes or even replacement of entire nucleotides by fluorophores. (B) Example strategies for preparing fluorescently labeled RNA for in vitro studies. Fluorescent labels are shown as red stars. $(C)$ Example strategies for preparing fluorescently labeled protein for in vitro studies. $(D)$ For intracellular single-molecule studies, RNA can be prepared using many of the methods suitable for in vitro work (described in $B$ ) and subsequently introduced into cells via microinjection or transfection. Alternatively, RNA transcripts can be labeled by inserting stem-loop repeat sequences that are bound by their cognate RNA-binding proteins, which are expressed as fusions with intrinsically fluorescent proteins (RBPFP). (E) Example strategies for preparing fluorescently labeled protein for intracellular studies. In contrast to RNA, there is greater overlap between strategies for labeling protein that are suitable for in vitro and intracellular work. A small number of strategies, such as click chemistry using strained alkynes, can be applied to both RNA and proteins. 
and/or in vitro transcribed segments (Solomatin and Herschlag 2009; Turunen et al. 2014).

In addition to chemical synthesis and ligation, other labeling techniques that hold promise for RNA labeling include position-specific labeling of RNA (PLOR), fluorogenic RNA aptamers, $2^{\prime}-5^{\prime}$ branched labeling, and engineered tRNA-modifying enzymes (Li et al. 2015; Carrocci et al. 2017; Trachman et al. 2017).

A simple alternative to covalent modification is to anneal the RNA of interest with one or more tightly binding complementary oligonucleotides, frequently DNA or locked nucleic acid (LNA) carrying a fluorescent label (Fig. 2B). The most prominent implementation of this strategy has been for studies involving large RNAs, especially the ribosome, in which a fluorescent oligonucleotide probe is annealed to short extensions engineered at various locations into the ribosomal RNA (Kim et al. 2014). In principle, such a probe can be designed for any RNA sequence, provided that the probe's hybridization is specific and only minimally perturbs the local and global RNA structure (Crawford et al. 2008; Rinaldi et al. 2016).

\subsubsection{Labeling Protein Components}

For proteins, fluorophore tags may be genetically appended, as is the case with fluorescent proteins (FPs) including green fluorescent protein (GFP), mCherry, and their many variants (Thorn 2017). However, FP tags are used almost exclusively in the context of intracellular studies (see Sec. 5.1) as their large size and unfavorable photophysical properties (e.g., propensity for blinking, low brightness) so far make them less desirable for in vitro single-molecule studies.

The shortcomings of FPs render proteins a more challenging target for site-specific labeling compared with RNA and the options for selective conjugation chemistries for amino acids are more limited compared with nucleotides. Fluorophore-NHS-ester derivatives readily react with primary amines and so can be used to label lysine residues as well as the amino terminus. For in vitro studies using recombinant protein, a more conventional strategy is to use fluorophore-maleimide derivatives, which have greater selectivity for the thiol side chain of rarer cysteine residues (Fig. 2C). This method typically requires that native cysteines be removed or significantly less reactive (and/or accessible) than the desired labeling position. Such was the case in a study of the role of initiation factor 2 (IF2) from Escherichia coli, in which removal of the three native cysteines in IF2 resulted in an unstable protein; however, labeling of an additional cysteine introduced through mutagenesis in the presence of the other native cysteines still resulted in site-specific labeling (Wang et al. 2015).
Among the other methods for site-selective labeling of proteins that have been developed (Adumeau et al. 2016), the use of unnatural amino acids to incorporate reactive handles, such as those for click chemistry, is one of the more promising avenues to achieve site specificity (Liu and Schultz 2010; Lee et al. 2016).

Purification of labeled protein from unincorporated fluorophore can be nontrivial because proteins, being more hydrophobic than nucleic acids, have a greater propensity to bind fluorescent dyes nonspecifically. The purification scheme therefore merits careful consideration. Although extensive dialysis or size-exclusion chromatography may be sufficient in some cases, for others unreacted fluorophore may be most robustly removed with ion-exchange chromatography under denaturing conditions (Hickerson et al. 2005).

Protein structure data are frequently unavailable and protein folding is difficult to predict. Furthermore, the complete details of a protein's RNA binding site and other interacting partners are frequently uncharacterized or illdefined, thus precluding the rational choice of a labeling position. Despite these gaps in knowledge, there are many cases in which determining the presence or absence of a given protein is sufficient, requiring only that the protein be fluorescently labeled in a way that preserves function. However, the knowledge of a fluorophore's exact location on the three-dimensional structure of a protein is required if the protein serves as the donor or acceptor in an smFRET experiment (see Sec. 3.1).

A third alternative for protein labeling are self-labeling protein tags and enzyme-mediated labeling tags that are genetically encoded. These tags have been developed to allow covalent attachment of small-molecule fluorescent dyes, which can be used to label proteins in live cells, in cell extracts, or to label recombinant proteins (Fig. 2C). Of these, notable examples include the SNAP and CLIP tags, based on human $O^{6}$-alkylguanine-DNA alkyltransferase, and the engineered dehalogenase HaloTag, all of which catalyze covalent self-addition of their respective ligands (Gautier et al. 2008; Los et al. 2008; Sun et al. 2011). A conceptually similar, albeit noncovalent, self-labeling tag is $E$. coli dihydrofolate reductase (eDHFR) (Calloway et al. 2007).

\subsubsection{Modification of Biomolecules for Surface Immobilization}

For certain types of SMFM, namely, TIRFM and ZMW, biomolecules of interest must be immobilized at the imaging surface. This is commonly achieved by conjugating the biomolecule of interest with biotin, which is then captured noncovalently by surface-bound streptavidin. Other noncovalent interactions, such as digoxigenin-antidigoxigenin 
antibody, as well as covalent interactions (e.g., thiol-maleimide or click chemistry) are also used for immobilization.

For short nucleic acids, biotin and digoxigenin can be incorporated during chemical synthesis, whereas the most straightforward strategy for longer RNA is to use biotinylated complementary oligonucleotides that stably hybridize to the RNA of interest, often in added $5^{\prime}$ or $3^{\prime}$ extensions. For RNA prepared by in vitro transcription using T7 RNA polymerase, modified nucleotides can be incorporated at the $5^{\prime}$ end of the transcript (Huang et al. 2008) as well as through enzymatic ligation (Kinoshita et al. 1997; Turunen et al. 2014) and the conjugation chemistries described above.

For modifying proteins, random biotinylation of lysines and cysteines with biotin $N$-hydroxysuccinimide esters and maleimides, respectively, provides the most direct path but with the least control over the site(s) of labeling. For recombinant proteins, the biotin ligase BirA can be used to catalyze the site-specific biotinylation of the 15-amino acid Avitag (Tirat et al. 2006). Biotin substrates for the self-labeling tags (i.e., SNAP, CLIP, and HaloTag) (see Sec. 2.2.2) are also commercially available. Last, surface-bound antibodies raised against a protein of interest have also been used to immobilize single proteins or large RNPs such as the spliceosome, as discussed below.

\section{APPLICATION EXAMPLE OF IN VITRO SINGLE- MOLECULE FLUORESCENCE: THE SPLICEOSOME}

The majority of in vitro SMFM studies can be broadly categorized into smFRET and colocalization experiments. In the following sections, we discuss these two types of experiments in the context of the yeast spliceosome. Rather than focusing solely on the biological insights into splicing provided by SMFM (for which we refer the reader to DeHaven et al. 2016), this section will highlight select studies drawn from the work of our group as well as others that illustrate the insights that SMFM provides with added emphasis on elements enabling its application.

\subsection{Single-Molecule FRET}

The spliceosome, which in all eukaryotes acts on precursor mRNAs (pre-mRNAs) to remove introns and join exons, is a challenging subject of study because of the sheer number of components involved: innumerable pre-mRNA substrates, five small nuclear RNAs (snRNAs), each bound to protein components to yield small nuclear ribonucleoproteins (snRNPs), as well as numerous, transiently associated splicing factors, resulting in a highly compositionally dynamic macromolecular machine (Fig. 3A). The associated, coordinated conformational changes that occur in the spliceosome, specifically those occurring in the distance range of $\sim 3-8 \mathrm{~nm}$, are best studied by smFRET. Although smFRET can be used to measure absolute distances, doing so with high accuracy is nontrivial, particularly when there is uncertainty about the assumed isotropic relative orientation of the two fluorophores on the RNA (Milas et al. 2013) and/or the possibility of protein-induced fluorescence enhancement (Hwang and Myong 2014; Lerner et al. 2016), and thus smFRET is most frequently used as a relative measure (dos Remedios and Moens 1995, McCann et al. 2010).

For splicing to occur with high fidelity, specific sequences that are often separated by hundreds of nucleotides in the pre-mRNA, namely, the $5^{\prime}$ and $3^{\prime}$ splice sites ( $5^{\prime}$ splice site [SS] and $3^{\prime}$ SS, respectively) as well as the branch site (BS), must be correctly identified by the splicing machinery and brought into close proximity, with the correct orientation and in the correct order, to permit the two isoenergetic transesterification reactions that the spliceosome catalyzes (Fig. 3A). To study pre-mRNA conformation, Abelson et al. (2010) labeled a model transcript based on the yeast Ubc4 pre-mRNA with a donor (Cy3, green star) and acceptor (Cy5, red star) fluorophore near the $5^{\prime}$ and $3^{\prime}$ SSs, respectively (Fig. 3B), and monitored pre-mRNA conformational changes using FRET. Even in buffer alone, the pre-mRNA molecules showed fluctuations between various FRET states, indicating that pre-mRNAs adopted different conformations over time. Point mutations at the $3^{\prime}$ SS had a significant impact on the conformations and dynamics of the RNA, underscoring the degree to which RNA sequence and structure are linked.

A follow-up study by Krishnan et al. (2013) used a similar smFRET strategy to examine the structural remodeling of the Ubc4 pre-mRNA in the context of a particular step of the splicing cycle, this time placing the donor and acceptor at the BS and $5^{\prime} \mathrm{SS}$, respectively. One general feature of in vitro experiments is a high degree of control over reaction conditions and components. To preclude the possibility of pre-mRNA conformational changes brought about by RNAbinding proteins present in WCE but unrelated to splicing, Krishnan et al. (2013) used an immunoprecipitation-based immobilization strategy termed single-molecule pull-down (SiMPull) FRET (Kahlscheuer et al. 2015). This allowed them to selectively isolate splicing complexes and stall those complexes at the $\mathrm{B}^{\text {act }}$ stage (Fig. 3C). Leveraging this immobilization strategy, combined with the selective reintroduction of additional recombinant splicing factors and ATP, allowed for characterization of pre-mRNA conformational dynamics by smFRET at a specific step of splicing.

Analysis of the donor and acceptor fluorescence signals from individual immobilized spliceosomal complexes begins with calculation of the relative FRET efficiency 
A

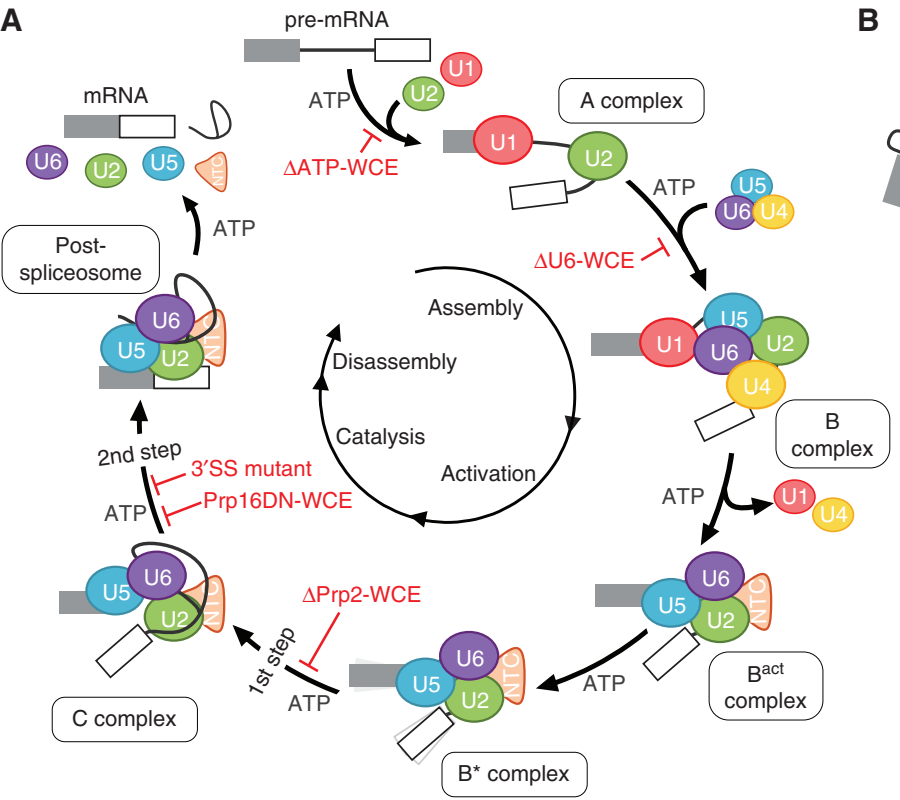

D

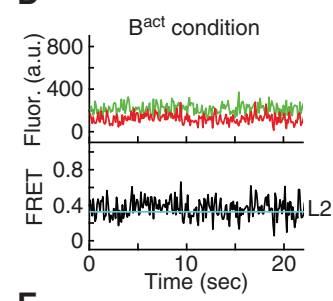

E
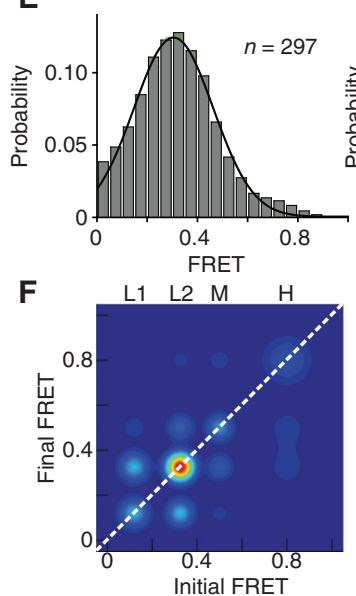
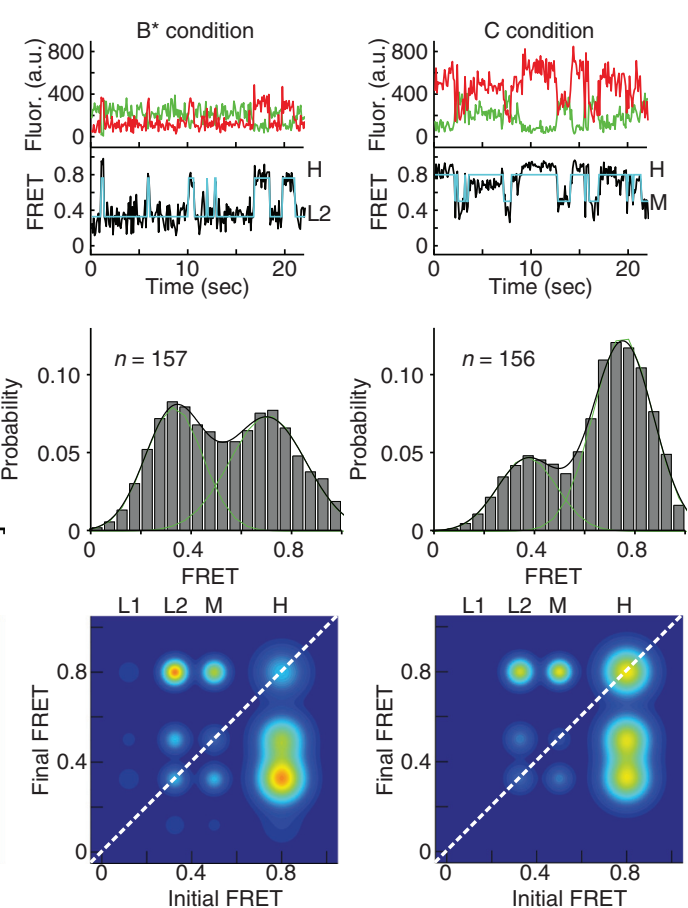

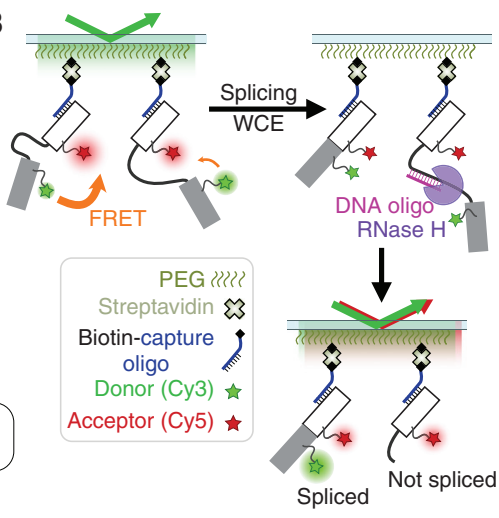

C

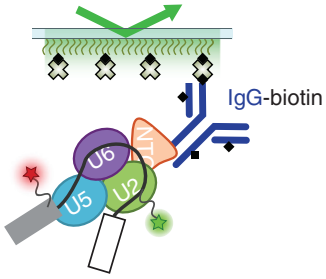

G
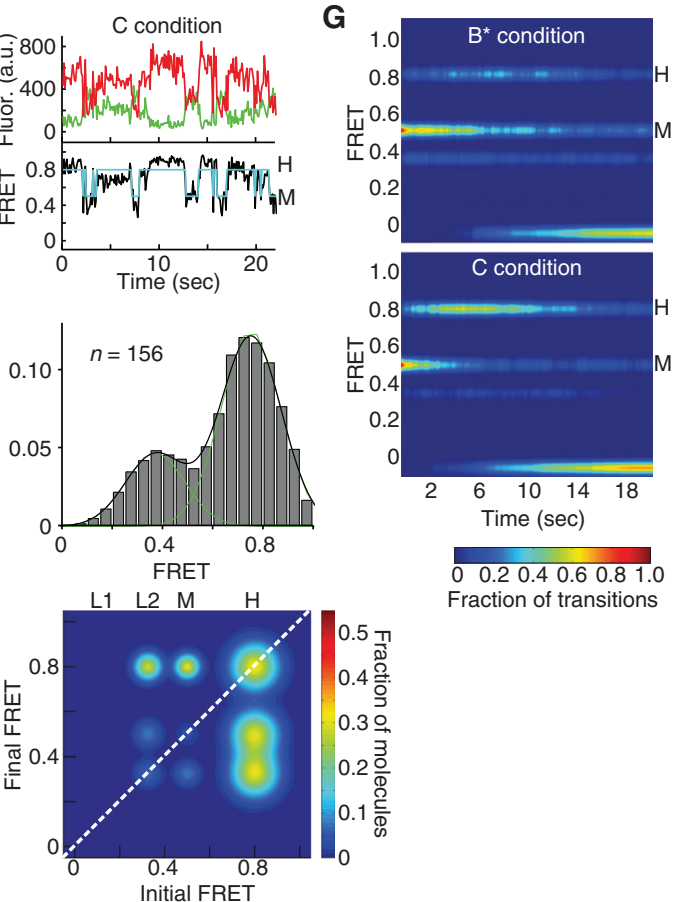

Fraction of transitions

Figure 3. Insights into yeast pre-mRNA conformational dynamics from in vitro single-molecule Förster resonance energy transfer (smFRET) studies. (A) Overview of the splicing cycle, in which the $5^{\prime}$ (gray) and $3^{\prime}$ (white) exons of the pre-messenger RNA (mRNA) are ligated and the intron (black line) is removed. Colored circles represent the various small nuclear ribonucleoproteins (snRNPs) required to catalyze splicing. Red annotations represent experimental conditions that can be used to block the cycle at various points. WCE, whole-cell extract. (B) Experimental design used by Abelson et al. (2010) to monitor conformational changes in a doubly labeled pre-mRNA construct through changes in FRET. An in situ RNase H assay, using a DNA oligonucleotide that hybridizes to sequences in the intron, verified that modifications made to the RNA to adapt it to single-molecule observation did not inhibit splicing. RNA molecules observed to have both fluorophores under direct excitation had been successfully spliced, whereas those showing only the acceptor fluorophore had not. (C) Experimental smFRET design from Krishnan et al. (2013) in which single-molecule pull-down (SiMPull) was used to isolate the $\mathrm{B}^{\text {act }}$ complex assembled on a pre-mRNA labeled near the branch site (BS) and 5' splice site (SS). (D) Example fluorescence-time traces from the donor (green) and acceptor (red) fluorophores attached to single pre-mRNA molecules under different experimental conditions designed to promote formation of the $\mathrm{B}^{\text {act }}, \mathrm{B}^{*}$, or $\mathrm{C}$ complexes, respectively. (Legend continues on following page.) 
(Fig. 3D) (Blanco and Walter 2010), which can then be used to create histograms of relative FRET efficiency that effectively represent the time-weighted conformational ensemble of molecules (Fig. 3E). Although the presence of multiple conformations is indicated by the presence of corresponding peaks, such histograms are largely unable to distinguish between molecules that show distinct kinetic behaviors, adopt varying numbers of states, or show overlapping, noise-broadened FRET distributions. Characterization of such nuance typically provides useful biological insight, particularly for highly reversible, multistep processes such as splicing, and requires appropriate analytical methods (Shi et al. 2008; Moffitt et al. 2010; Grima et al. 2014; Colomb and Sarkar 2015). smFRET time traces are often fit using hidden Markov models (HMMs) to idealize noisy and continuous FRET efficiency time-series data into a set of discrete states that facilitate the extraction of kinetic information (Blanco and Walter 2010; Liu et al. 2010). Fractions of molecules that undergo specific conformational changes, defined by a particular initial and final idealized FRET efficiency, can be identified using transition occupancy density plots (TODPs). This method was used to survey changes in the dynamic behavior of splicing complexes stalled at specific points in the splicing cycle (Fig. 3F) (Krishnan et al. 2013).

Although the catalytically active $B^{*}$ complex can undergo the first chemical reaction of splicing, this step is greatly enhanced by Cwc25, a protein known to be associated with the nineteen complex (NTC). Cwc25 is known to bias the spliceosome toward a configuration in which the $5^{\prime} \mathrm{SS}$ and BS are in close proximity by slowing the reverse reaction. This effect was directly visualized in this study using another common single-molecule analysis tool known as a postsynchronized histogram (Fig. 3G), where molecules starting in a medium FRET state fluctuate frequently between $\mathrm{H}$ and $\mathrm{M}$ states, but primarily transition to and stay in the $\mathrm{H}$ state in the presence of Cwc25. Cwc25 was thus found to act as a "pawl" to stabilize the otherwise thermally "ratch- eting" pre-mRNA in its proximal, active conformation, promoting the transition to the $\mathrm{C}$ complex accompanied by the first chemical reactions of splicing.

The conclusions from Krishnan et al. (2013) about premRNA conformational remodeling and dynamics, some of which are highly reversible, and the role of splicing factors in activating the spliceosome were in good agreement with complementary studies by Crawford et al. (2013), who combined observables from smFRET with colocalization single-molecule spectroscopy (CoSMoS) to correlate premRNA conformational changes observed through smFRET with specific spliceosome compositions by monitoring colocalization of U1 and the NTC.

\subsection{Single-Molecule Colocalization}

In CoSMoS, multiple biological components are fluorescently labeled with spectrally distinct fluorophores and monitored simultaneously. The ability to observe multiple species provides a direct method of discriminating between parallel pathways that use alternative macromolecular assemblies of largely similar, but not identical, composition. Because of the binary nature of binding (i.e., either bound or unbound), rapid and large changes in fluorescence signal intensity are observed on binding and dissociation of a fluorescent molecule or complex, which can be used to yield kinetic information. Furthermore, coincidence of two fluorescence signals indicates the simultaneous presence of differentially labeled components.

In a series of multicolor CoSMoS experiments, Shcherbakova et al. (2013) examined the order of assembly of the U1 and U2 snRNPs on the 5'SS and BS for a number of pre-mRNAs that show different in vitro splicing efficiencies (Fig. 4A). Using genetically modified WCE from yeast that contained SNAP-, CLIP-, and eDHFR-tagged protein components, they prepared splicing-active extracts containing U1, U2, and U5 snRNPs where each was labeled with a unique fluorophore. Fluo-

\begin{abstract}
Figure 3. (Continued) The FRET efficiency (black) is fit using hidden Markov modeling (HMM, cyan line) to identify unique FRET states. Fluor., fluorescence intensity; a.u., arbitrary units. (E) Histograms of FRET efficiency, generated here by combining the first 100 movie frames from each molecule in a given experimental condition, can be used to identify underlying FRET states. $(F)$ Transition occupancy density plots (TODPs) showing the fraction of molecules in a given experimental condition that show transitions between a given initial and final FRET state. A molecule that remains in the same state during the entire observation period, such as the one shown in $\mathrm{B}^{\text {act }}$ condition in panel $D$, contributes to peaks on the diagonal. As splicing progresses from $\mathrm{B}^{\text {act }}$ to $\mathrm{B}^{*}$ to $\mathrm{C}$, there is an increase in the number of transitions to the highest FRET states (H), in which the 5'SS and BS are in close proximity. The fraction of molecules that stably reside in the high FRET state is greatest under $\mathrm{C}$ complex conditions, which is consistent with molecules that have undergone the first step of splicing. $(G)$ Postsynchronized histograms of molecules collected under B* and C complex conditions were plotted such that they are synchronized to start in state M. Molecules reversibly transition between $\mathrm{M}$ and $\mathrm{H}$ states under $\mathrm{B}^{*}$ conditions. In contrast, under $\mathrm{C}$ complex conditions (in which Cwc25 is present), molecules preferentially transition from $\mathrm{M}$ to $\mathrm{H}$, where they largely reside until photobleaching (FRET values below zero). ( $D-G$ adapted, with permission, from Krishnan et al. 2013.)
\end{abstract}


A.P. Jalihal et al.
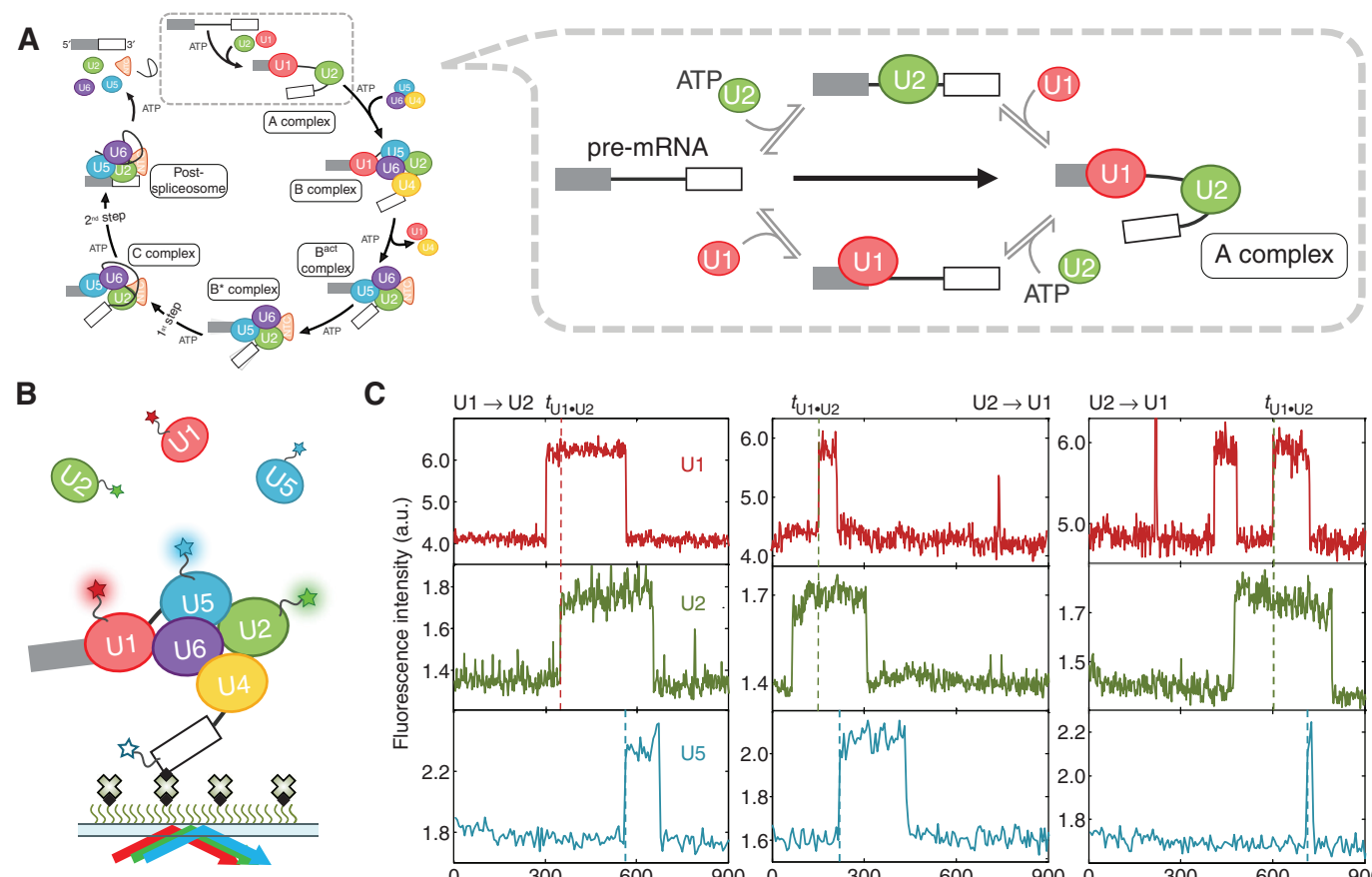

C

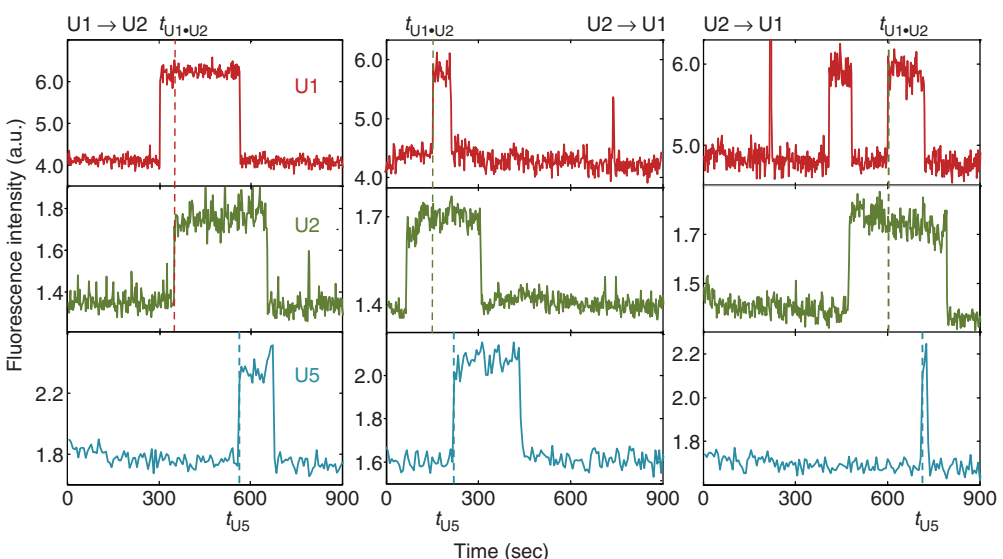

D

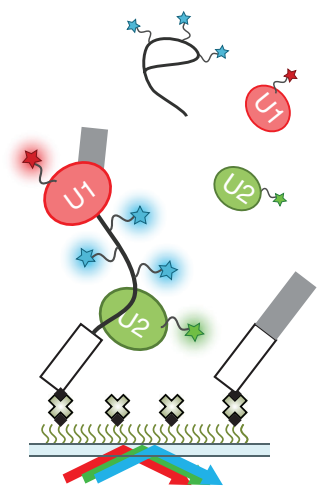

E

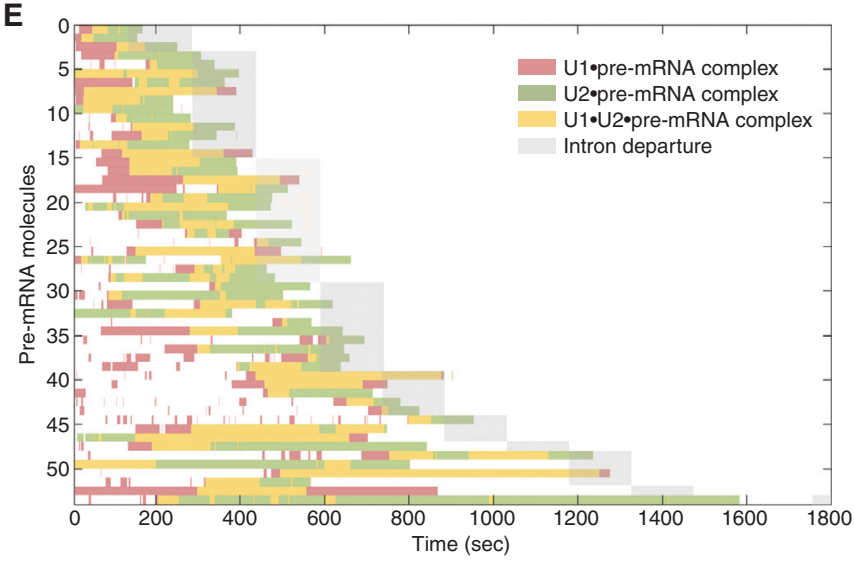

Figure 4. Insights into early spliceosome assembly in yeast from in vitro single-molecule fluorescence colocalization studies. (A) Detailed view of the early steps of yeast spliceosome assembly in context of the larger splicing cycle. In the earliest steps of splicing leading to formation of the A complex, the U1 and U2 small nuclear ribonucleoproteins (snRNPs) assemble on the pre-mRNA near the $5^{\prime}$ splice site (SS) and branch site (BS), respectively. (B) Experimental design used by Shcherbakova et al. (2013) to study the order of spliceosome assembly by simultaneously monitoring the arrival of fluorescently labeled U1, U2, and U5 snRNPs in wholecell extracts (WCEs). The locations of the surface immobilized pre-mRNA were established at the beginning of the experiment using a fluorophore incorporated into the $3^{\prime}$ exon, which was subsequently photobleached (white star) before the introduction of WCE containing labeled snRNPs. (C) Example fluorescence-time traces for three premRNA molecules, showing instances in which U1 arrival preceded arrival of U2 (left) or where U2 preceded U1 (center, right). The sudden increase in fluorescence in a given channel indicates the binding and stable association of the respective snRNP. Only pre-mRNA molecules that ultimately showed the arrival of U5, and thus progression to the B complex, were considered for analysis. (D) Experimental design used by Shcherbakova et al. (2013) to monitor the binding and dissociation of labeled U1 and U2 on a pre-mRNA containing multiple fluorophore in the intron. The high number of fluorophores (approximately eight) in the intron prolonged the viable observation window, as well as allowed dissociation of the intro lariat (sharp loss of all blue fluorescence) to be differentiated from photobleaching (stepwise loss of blue fluorescence). (E) Rastergram showing the residence time of $\mathrm{U} 1$ and $\mathrm{U} 2$, as well as the time until intron departure for 54 pre-mRNA molecules. (C,E reproduced, with permission, from Shcherbakova et al. 2013 and Elsevier.) 
rescence of these snRNPs was then monitored at previously established locations of immobilized pre-mRNA molecules (Fig. 4B).

A key strength of CoSMoS is the fact that observation of one fluorophore-labeled component can indicate the presence of one or more secondary components that are constitutively present in the complex. By examining many multichannel fluorescence time traces, Shcherbakova et al. (2013) observed that the preferred order of binding of U1 and U2 varies for different pre-mRNAs (Fig. 4C). Further, the investigators found evidence that traces proceeding through the U2-first pathway did not represent dead-end complexes because they observed subsequent recruitment of U5 as a marker for the next splicing step. This ability to discern molecules traversing a particular reaction pathway within a highly heterogeneous reaction mixture, a hallmark of single-molecule methods, is particularly useful in a field such as splicing where the in vitro splicing efficiency of premRNAs in crude cell extracts varies greatly, typically between $20 \%$ and $80 \%$.

Shcherbakova et al. (2013) were also able to examine whether the order of U1 and U2 assembly affected the ability to advance through eventual intron removal by labeling U1, U2, and the intron of the pre-mRNA (Fig. 4D). They observed that molecules following U1-first or U2-first paths proceeded through the second step of splicing equally well. They also found that the interaction of U1 with the pre-mRNA was more dynamic (i.e., showed more frequent binding and dissociation) than that of U2, and neither snRNP bound after intron loss (Fig. 4E).

Given the relatively long timescales of splicing in vitro, photobleaching of fluorophores poses an obvious constraint in single-molecule fluorescence imaging. Intermittent laser illumination and a high number of fluorophores incorporated into the intron can prolong the observation window in CoSMoS and help distinguish true dissociation from photobleaching. This general strategy is frequently applied to RNP studies when loss of a component from a complex is of interest-for example, in Argonaute (Ago)mediated RNA slicing (Salomon et al. 2015) - and is important for high-background specimens as encountered in intracellular studies (see Sec. 5.1).

In addition to identifying components of a complex and characterizing their binding and/or dissociation kinetics, the precise identity of a molecule or changes in its structural state can also be inferred from the binding/dissociation kinetics of a dynamic, state-specific reader probe (Johnson-Buck et al. 2015; Rinaldi et al. 2016). Taken together, these approaches exemplify the strengths of single-molecule microscopy for studying the interplay between multiple biological components.

\subsection{Advanced Single-Molecule Analysis}

Perhaps one of the most challenging aspects of studying complex RNPs using single-molecule experiments is determining how best to analyze the data to fully extract the details of interactions given that the entire scope of molecular behaviors is directly observed (within the experimental limit of accessible spatiotemporal resolution). Although strategies such as selective labeling and SiMPull-based enrichment help reduce the data complexity and focus the experiment on observables of interest, single-molecule fluorescence traces are frequently very feature-rich and heterogeneous in a system-specific manner, motivating continuous innovations in data analysis.

For example, Blanco et al. (2015) used the Ubc4 premRNA labeled for smFRET at the 5'SS and BS and collected data from more than 10,000 molecules at three time points and under a variety of conditions designed to stall splicing at various steps (Fig. 3A, red annotations). To examine premRNA dynamics during splicing in greater detail, facilitate finding commonalities among many idealized smFRET traces, and compare properties across a variety of experimental conditions, these investigators developed singlemolecule cluster analysis (SiMCAn) (Blanco et al. 2015). In SiMCAn, each trace is represented by a FRET similarity matrix (FSM) that describes the occupancy of FRET states and the kinetics of transitions between these states. FSMs for all traces from one or more experimental conditions were then clustered based on their similarity (Fig. 5A), allowing individual molecules to be segregated on the basis of both their FRET states and kinetics. The result was a set of 35 clusters, 25 and 10 showing dynamic and static FRET, respectively, representing the conformational behaviors observed across all conditions in the large data set.

A second round of clustering was then performed to determine the prevalence of particular conformational behaviors. This grouping revealed behavioral trends along the splicing cycle. For example, clade VII was found to be comprised of a group of clusters typifying FRET states that correspond to a pre-mRNA conformation in which the $5^{\prime} \mathrm{SS}$ and BS are brought into close proximity (Fig. 5B). This singlemolecule behavior was observed in either wild-type (WT) or mutated 3'SS pre-mRNA when using WCEs containing a dominant-negative form of the helicase Prp16 (Prp16DN), a mutant that is known to stall splicing just before the second chemical step, which is indeed expected to lead to the observed proximity between $5^{\prime}$ SS and BS. That SiMCAn also can discover new states was shown by the observation of a time-dependent increase in the separation of the $5^{\prime} \mathrm{SS}$ and BS (reflected by increased occupancy in clade I, a static lowFRET state) for specifically the 3'SS mutant when splicing was allowed to progress in the presence of WT Prp16. This 
A.P. Jalihal et al.

A
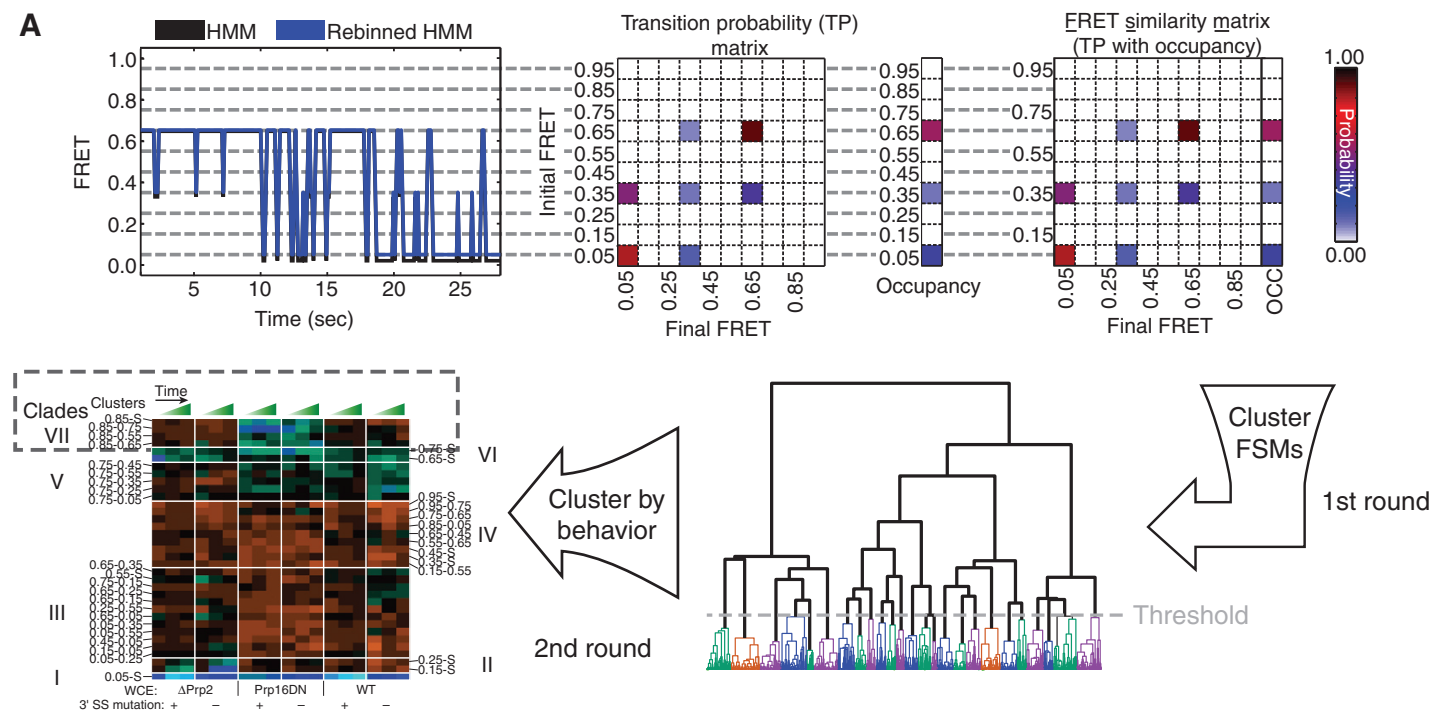

B

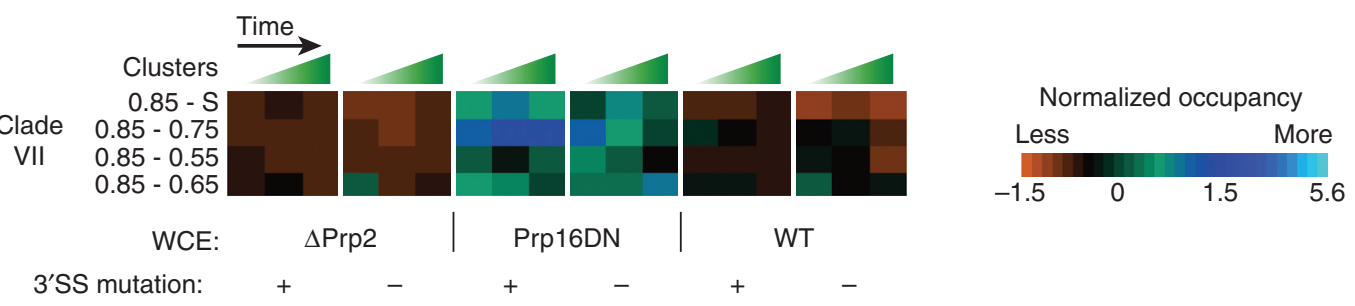

Figure 5. Advanced analysis of single-molecule Förster resonance energy transfer (smFRET) trajectory data-singlemolecule cluster analysis (SiMCAn). (A) Workflow for SiMCAn. smFRET traces idealized with hidden Markov modeling (HMM) are rebinned to a discrete set of FRET values and then converted to a transition probability (TP) matrix, which describes the probability of observing transitions between various FRET states. The TP matrix for each molecule is combined with an additional occupancy column that describes the time spent in a given FRET state, yielding a FRET similarity matrix (FSM) for each molecule. The set of FSMs from all molecules are then clustered to identify common behaviors (clusters). The fractional representation of molecules from each experimental condition in a given cluster is then used in a second round of clustering to uncover particular behaviors (clusters) that are overor underrepresented in a consistent way across a set of various experimental conditions. $(B)$ Detailed view of clade VII. Experiments were performed using either wild-type (WT) yeast whole-cell extracts (WCEs), WCEs lacking Prp2, or WCEs containing a dominant-negative form of Prp16 (Prp16DN), on splicing complexes assembled on WT Ubc4 pre-messenger RNA (mRNA), or Ubc4 pre-mRNA containing a mutated 3's splice site (SS). (Adapted, with permission, from Blanco et al. 2015.)

increased separation suggested a late-stage proofreading step involving Prp16 to allow for correct selection of the 3'SS, a finding subsequently characterized in greater detail by Semlow et al. (2016). SiMCAn thus facilitates comparison of data-rich single-molecule data sets to quickly identify unique behaviors that provide new biological insight.

\section{DEEPER CONSIDERATIONS FOR STUDYING SINGLE RNPS IN VITRO}

As the selected studies of the spliceosome described above show, RNPs can be highly dynamic and show heterogeneous behavior (Wahl et al. 2009). Although the biological process of interest can often be recapitulated in vitro, there is a risk of losing the "true" diversity, specificity, and speed of molecular behaviors that exist inside living cells. To further understand the scope and limitations of in vitro singlemolecule studies of RNPs, in particular, some important design considerations for TIRFM-based imaging, the most widely used in vitro single-molecule imaging method, are discussed in the following.

The first consideration in designing any biophysical experiment to study RNPs is sample preparation. To minimize the variables influencing the process under study, in vitro single-molecule studies use minimal mixtures of purified RNAs and proteins. However, reactions involving RNPs often engage multiple, dynamically exchanging components whose stoichiometry is sometimes poorly defined. 
In such cases, it may be advantageous to instead isolate "native" holoenzyme complexes from whole cell lysates to recapitulate biological activity. This is exemplified by the strategy used by Krishnan et al. (2013), in which SiMPull was used to isolate the $\mathrm{B}^{\text {act }}$ complex. The drawback of this strategy for sample preparation is that the composition and stoichiometry of the isolated, individual complexes is undetermined. Importantly, molecular complexes obtained by native isolation and reconstitution may differ functionally. In some cases, such a discrepancy in activity can yield important biological insights, as single-molecule observations of the human telomerase complex illustrated ( $\mathrm{Wu}$ et al. 2015). This RNP consists of the RNA-templated enzyme TERT, which adds repetitive nucleotide sequences to telomere ends in template-dependent manner. In vitro reconstituted minimal TERT RNPs were found to be monomers that were functionally active when assayed for repeat addition. In contrast, native holoenzymes isolated from cell lysates were found to exist as dimers and higher-order multimers, and showed lower repeat addition activity, pointing at intrinsic regulatory mechanisms ( $\mathrm{Wu}$ et al. 2015). Additionally, native holoenzyme purification is often challenging and is not always conducive to facile fluorescent labeling.

The second consideration of SMFM experiments relates to the timescales of the reaction under study. To obtain useful statistics on reversible processes, such as those involving conformational changes or binding, it is important to be able to monitor a single molecule/complex over multiple reaction cycles, to adequately probe the residence times in each state (Moffitt et al. 2010). By design, TIRFM experiments extend observation windows for single molecules by anchoring them to a slide surface. Immobilization close to the surface, however, can occasionally introduce artifacts because of the effects of surface interactions on molecular conformation and folding kinetics (Yasuda et al. 2003). These effects can be overcome by using alternative immobilization strategies, such as encapsulating individual molecules or complexes in lipid vesicles that, in turn, are tethered to the surface (Okumus et al. 2004). Such alternate strategies, however, serve as practical solutions only when specific constraints or limitations are encountered on standard TIRFM-based imaging.

Given the compositional complexity of RNPs and their sensitivity to reaction conditions, it is arguably most informative to study them in their native context, in vivo. Observations of single-molecule interactions in living cells include the full context of biological pathways, arising from dynamic changes in RNP composition, effects of molecular chaperoning, molecular crowding and spatiotemporal organization. Using living cells themselves as reaction vessels to study RNPs trades precise control over the reaction condi- tions for new dimensions in single-molecule behavior. Studying the behavior of single RNPs in their cellular context represents a new paradigm for studying biology that harnesses the power and versatility of SMFM to unravel the roles of RNPs in shaping diverse cellular phenotypes.

\section{SINGLE-RNP VISUALIZATION IN LIVING CELLS}

The toolbox of complementary fluorescence-based approaches available today allows dynamic RNP interactions to be probed directly inside living cells. For example, fluorescence recovery after photobleaching (FRAP) and FCS both quantify diffusion of labeled molecules (McNally 2008; Bacia et al. 2014). The former measures the rate at which fluorophore-labeled molecules diffuse back into a small photobleached area (typically over minutes), whereas the latter measures fluorescence fluctuations in a small volume of the cell on subsecond timescales. These techniques have been used to study fluorescent molecules inside living cells for more than three decades, and although they do not necessarily provide single-molecule resolution, they have set the stage for intracellular SMFM.

More recently, the commercialization of photostable fluorophores, increasingly sensitive cameras, and super-resolution illumination strategies together have contributed to advances in a third technique, intracellular single-particle tracking (SPT) (Shen et al. 2017). SPT bridges the capabilities of ensemble approaches such as FRAP and FCS (diffusion information) with those of in vitro single-molecule analysis, such as colocalization (interaction) time and stepwise photobleaching analysis (for stoichiometry).

The principle behind SPT is that fluorescently labeled molecules, when present at sufficiently low densities, can be detected and tracked in time as bright, diffraction-limited "spots" using an illumination strategy such as HILO or TIRFM. The resulting trajectories represent the actual diffusive motion of the molecules and can reveal transient interactions with their local environments. Thus, intracellular SPT can be applied to study RNP reactions using cultured living cells themselves as reaction vessels, providing exquisite spatiotemporal resolution to make observations that enable both hypothesis- and discovery-driven approaches to studying the biophysical behavior of RNPs (Fig. 6).

The following sections briefly review methods for the fluorophore labeling and intracellular macromolecule delivery needed to perform intracellular SPT.

\subsection{Fluorescent Labeling Strategies Compatible with Intracellular RNP Detection}

One advantage of using living cells in fluorescence microscopy is the ability to genetically encode fluorophore tags in 
A.P. Jalihal et al.

A

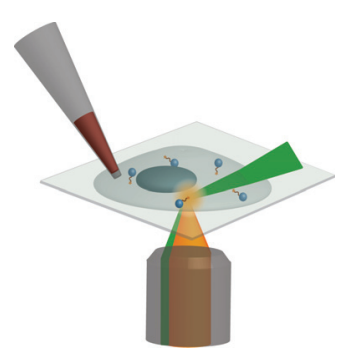

C

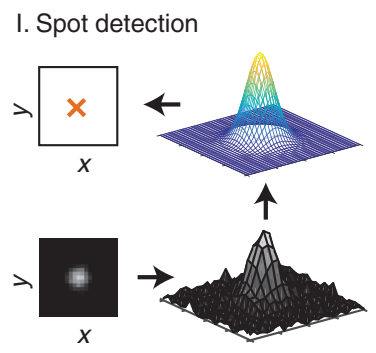

D

I. Photobleaching trace II. Step counting

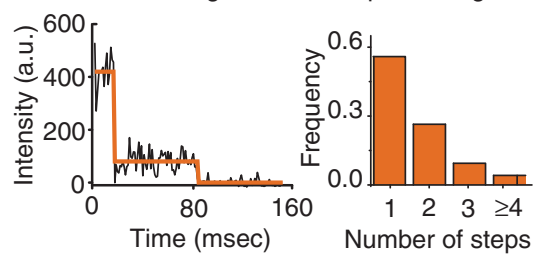

B

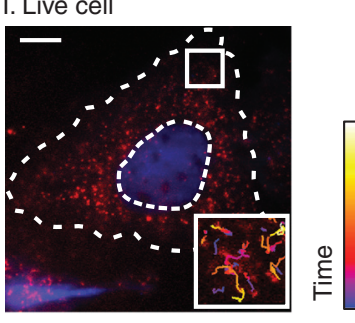

II. Particle tracking

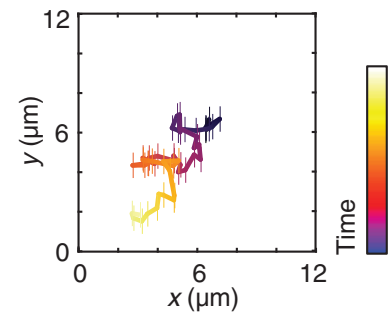

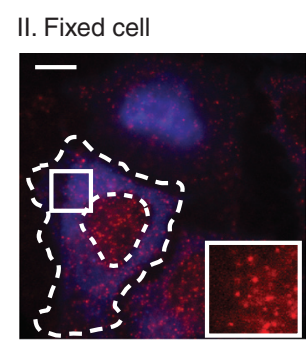

III. Diffusion analysis

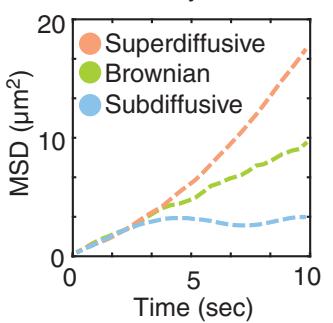

E

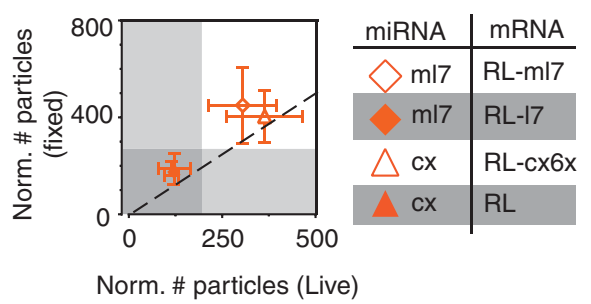

Figure 6. Intracellular single-molecule visualization. (A) Microinjection is an effective strategy to deliver labeled RNAs into living cells. The injected material can be imaged using various fluorescence microscopy techniques. Intracellular single-molecule high-resolution localization and counting (iSHiRLoC) uses highly inclined and laminated optical sheet (HILO) illumination to rapidly acquire images with single-molecule resolution for particle tracking. (B) Injected cells are distinguished from noninjected cells by the presence of an injection marker (a slowly diffusing, fluorescently labeled inert compound). Shown here are two cells injected into the nucleus (I) and cytosol (II) with Alexa488-labeled 100-kDa dextran. Labeled microRNAs (miRNAs) appear as diffusing, diffraction-limited spots on time-lapse imaging of live cells (I, inset) or as diffraction-limited spots in formaldehyde-fixed samples (II, inset). Scale bars, $10 \mu \mathrm{m}$. (C) (I) The intensity profiles of diffraction-limited spots, recorded as pixelated point-spread functions, can be analyzed to obtain the spot centers with subpixel accuracy, such as by fitting the intensity profile to two-dimensional (2D) Gaussians. (II) Particle-tracking of the spot centers results in 2D or 3D diffusion trajectories. (III) Displacements in successive frames yield mean-squared displacement (MSD) profiles, informing about diffusion types. (D) Photobleaching analysis. Cy5 fluorophores bleach rapidly and in a stepwise manner. The number of discrete step drops in intensity is a proxy for the number of molecules in a single diffraction-limited spot. (E) Correlating the number of fluorescent spots in fixed tissue with the number of diffusion-filtered trajectories yields an estimate of turnover and functionality of the labeled molecules. The open shapes represent counts of a mutant (mutant let-7, ml7) and artificial ( $c x c r 4, c x)$ miRNA coinjected with messenger RNAs (mRNAs) bearing complementary binding sites, and the filled shapes represent conditions in which the coinjected mRNAs bore mismatched (let-7,17) or no binding sites in the $3^{\prime}$ untranslated region (UTR) downstream from an open reading frame (ORF) encoding Renilla luciferase. ( $D$, Adapted, with permission, from Pitchiaya et al. 2012; $E$, adapted, with permission, from the authors and John Wiley.)

the form of FPs. The most widely adopted intracellular RNA-labeling strategy has been the use of RNA-binding proteins (RBPs) fused with an FP (Tyagi 2009). This method, first demonstrated for single-RNA detection by Robert Singer's group, exploits the high specificity and affinity with which RBPs such as the viral coat proteins from the MS2 or
PP7 phages bind their cognate RNA stem-loop structures. Since then, this technology has been expanded to other bacteriophages, such as the $\lambda_{\mathrm{N}}$ protein binding to the RNA stem loop of the boxB gene. Labeling is achieved by inserting these stem-loop sequences into untranslated regions (usually the $3^{\prime}$ UTRs) of mRNA transcripts, which are 
then bound by their respective RBP fused to an FP (Fig. 2D). Because the excess of unbound RBP-FP fusion protein creates a high fluorescence background, and because photobleaching is vastly accelerated in live cells as compared with in vitro, multiple copies of the stem-loop sequence (typically 8 to 96 ) are required for single-molecule sensitivity. Furthermore, multiple transcript species within the same cell can be simultaneously monitored by using combinations of RBPs tagged with distinct FPs (Hocine et al. 2013).

The biggest limitation of RBP-based detection is that their cognate stem loops are $\sim 20$ nucleotides long, which makes RBP-based methods unsuitable to study small noncoding RNAs such as miRNAs, small interfering RNAs (siRNAs), tRNAs, etc. whose function is sensitive to RNA length. Furthermore, the added molecular mass of bound RBP-FP fusion proteins can alter the diffusion properties, localization, and turnover of RNPs.

Another limitation of FPs is their relatively long maturation time. For studies specifically probing protein translation, using a classical fluorescent protein to study translation rate is of limited use because of the slow maturation of the FP (the fastest maturing GFP variants fold in $\sim 10 \mathrm{~min}$ ) relative to translation elongation (seconds to minutes for a given transcript) (Thorn 2017). However, moving the fluorescent tag to an antibody probe that can recognize a peptide epitope with specificity as it emerges from the ribosome's exit tunnel allows rapid processes such as translation elongation to be studied in real time at singlemolecule resolution. Such fluorescent "immunolabeling" of proteins has been shown with the SunTag epitope and "spaghetti monster" fluorescent proteins (Fig. 2E). In these methods, single proteins are detected when multiple labeled antibody probes bind to a repeated epitope, in which detection is limited by diffusion of the probes and antibodyantigen affinity (Morisaki et al. 2016; Yan et al. 2016).

The relative simplicity of working with transiently or stably transfected cell lines has led genetically encoded FP methods for single-RNP visualization to become widely accepted. We refer the reader to reviews that cover the broad applications of RBP-based mRNA detection and live cell immunolabeling for intracellular single-molecule visualization (Buxbaum et al. 2015; Lyon and Stasevich 2017). An alternative approach to fluorescently label proteins uses self-modifying enzymatic tags (see Sec. 2.2.2 and Fig. 2E). Pulse labeling with a cell-permeable, fluorescent HaloTag substrate, for example, allows a sufficiently sparse set of the native proteins to be labeled. Schmidt et al. (2016) used this strategy to show that the telomerase RNP is dynamically recruited to the telomere ends of chromosomes.

An alternative RNP-labeling strategy that avoids genetic modification of RNAs/proteins for intracellular detection is to covalently attach synthetic fluorophore dyes to an ex- ogenous RNA and subsequently deliver it into cells. For longer RNAs, click chemistry has been used to covalently attach fluorophores to modified nucleotides that are cotranscriptionally incorporated into in vitro transcribed RNA without the need for lengthy exogenous sequence motifs (compare Fig. 2B and D). For eukaryotic mRNAs, fluorophores can be incorporated into mRNAs in the cap structure (Mamot et al. 2017), randomly incorporated throughout the transcript (Schulz and Rentmeister 2014), in the poly(A) tail, or between the $3^{\prime}$ UTR and poly(A) tail (Custer and Walter 2017). Such covalent attachment of the fluorophore allows labeled molecules to be detected with greater sensitivity, without the background of free fluorophores that is a recurrent challenge of RBP-based RNA detection. As with RBP-mediated labeling, however, there can be functional consequences to these covalent labeling strategies; for example, random fluorophore incorporation throughout the transcript was shown to dramatically impair the coding function, and incorporation of fluorophores in the $3^{\prime}$ UTR was shown to stabilize mRNAs (Custer and Walter 2017).

The choice of delivery method determines the types of observations that can be performed after exogenous chemical labeling. The following section briefly surveys these methods.

\subsection{Intracellular Delivery of Materials Labeled In Vitro}

The wide array of available intracellular delivery methods presents several choices for RNA and/or protein delivery (Stewart et al. 2016). They fall on a spectrum defined by, on one hand, their ability to deliver precise amounts of material to individual cells and by, on the other hand, their throughput of cells. One subset of delivery strategies aims to temporarily disrupt the cell membrane using detergents, bacterial pore-forming toxins, or various physical disruption methods (bead loading, fluid shearing, electroporation, etc.), relying on diffusion of cargo along concentration gradients and the ability of cells to reseal breaks in their cell membrane. These strategies achieve efficient cargo delivery but are not suitable for applications requiring precise dosage control. Another delivery strategy in this class involves vesicle carriers and relies on fusion of lipid vesicles with the cell membrane or natural endocytic processes for cargo delivery. Lipid-based transfection is perhaps the most widely used strategy; however, it is unsuitable for RNA delivery for single-molecule applications as the cargo often remains trapped in endosomes and is only slowly released into the cell (Hirsch and Helm 2015), leading to the risk of artifacts and/or misinterpretation.

In contrast, methods that involve active forces, such as delivery by direct microinjection using microneedles or 
A.P. Jalihal et al.

nanostraws, offer more precise control over the amount of material delivered to each cell, however, they can do so only with lower throughput. Microinjection, like other membrane disruption methods, relies on the ability of cells to rapidly reseal breaks in the cell membrane (Fig. 6A) and thus can encounter problems with cell viability if improperly executed (Pitchiaya et al. 2013). The microinjector needle is a fine glass capillary used to inject femtoliters of cargo solution into each cell, allowing for precise delivery into subcellular compartments such as the nucleus or the cytosol. Microinjection requires only small cargo quantities compared with any other bulk delivery strategy, making it a particularly valuable and preferred tool for delivering fluorescently labeled RNP components that may be technically challenging to label and purify.

\subsection{Analysis and Interpretation of SPT Experiments}

The first step for particle tracking is detection of the "spot" that represents the spatial location of a fluorescently labeled molecule of interest. The intensity levels of the brightest pixels of the spot together with the surrounding pixels approximate the point spread function (PSF) of the fluorophore(s) present on the molecule or complex. The challenge of spot detection lies in finding the center of the PSF, given the discrete pixel size and variable camera noise. A number of different algorithms exist today to perform this task (Fig. 6B) (Chenouard et al. 2014). Once detected, the spots can then be tracked (i.e., temporally linked) through successive frames to yield spatiotemporal trajectories, provided the spots are sufficiently visible in the focal plane in (almost) every frame, and the particles are present at sufficiently low density for the temporal linking to be unambiguous.

From these particle trajectories, the type of motion shown by individual molecules can be classified into one of various types of diffusion. The most common, simple, and useful tool to discriminate diffusive behaviors of single particles is the time-averaged mean-squared displacement (MSD) (Fig. 6C). This plot is used to characterize the trend of the displacement of the particle at increasing time intervals. For a given trajectory, the deviation of the MSD plot from linearity is used to classify the motion as diffusive (no deviation), subdiffusive (curved down), or superdiffusive (curved up). RNPs often show complex trajectories that can be modeled as mixtures of directed motion (such as during periods of active transport along axons) and Brownian (random) diffusion (Monnier et al. 2015). Together, such characterization can yield information regarding the molecular weight of the particle under study, and its interaction with its local environment, such as binding to and dissociation from unlabeled (and thus invisible) partners of distinct diffusivity (Knight et al. 2015).

Multicolor particle tracking can reveal even more about the intracellular milieu. By labeling molecular species using dyes with spectrally separated emission maxima, currently up to four different types of particles can be simultaneously tracked (Juette et al. 2014). The interaction between these differently labeled components can be read out as spatiotemporal colocalizations between trajectories of differently colored particles, similar to the in vitro techniques summarized in Section 3.2. Colocalizing trajectories can then be analyzed to characterize the kinetics and search dynamics of intracellular interactions, as has been the case for examining the intracellular search dynamics of RNP machines such as the Cas9 enzyme (Knight et al. 2015), telomerase (Schmidt et al. 2016), and miRNA-RISC (miRISC) complexes, as well as for studying the recruitment of small RNAs to sites of DNA double-strand breaks (Michelini et al. 2017).

The following section examines the example of intracellular single-molecule methods applied to the RNA silencing pathway.

\section{APPLICATION EXAMPLE OF IN VIVO SINGLE- MOLECULE FLUORESCENCE: RNA SILENCING}

The first reports of in situ single-miRNA visualization used complementary LNA hybridization probes to detect miRNAs in fixed cells and were applied to detect the subcellular localization and cell-to-cell variability in expression levels of miRNAs (Lu and Tsourkas 2009). Complementary ensemble-level observation of dynamic changes in subcellular localization of siRNA in living cells was reported by Ohrt et al. (2006). Examining the diffusion of chemically labeled siRNA strands using FCS, they found that the fluorophore labeling position on the double-stranded siRNA influenced intracellular diffusion and localization. Instead of using various commonly used lipid- and peptide-based transfection techniques, which can also influence siRNA localization (Chiu et al. 2004), the investigators used microinjection to deliver labeled, double-stranded siRNA variants directly into the cell. Microinjection also permitted them to start imaging as early as $10 \mathrm{~min}$ after introduction of the siRNAs, allowing them to study early steps of the siRNA pathway. Their observations corroborated earlier findings that the loading of the siRNA strand into RISC is sensitive to modifications at the $5^{\prime}$ end and overhangs of the guide strand. Observing the codiffusion of labeled siRNAs with GFP-tagged Argonaute protein using two-color FCCS, these investigators were able to compare the nuclear export pathways of "naked" and RISC-loaded siRNAs. FCS-based measurements of the molecular weights of nuclear and cytoplasmic RISC suggested that the two complexes are 
largely mRNA-bound and -unbound, respectively. By coinjecting labeled siRNA with purified mRNA target, they concluded that nuclear import of RISC is target-dependent (Ohrt et al. 2008).

Although FCS provides useful insight into diffusive processes and the concentration of fluorophores in a cell, it is limited by its confocal imaging geometry that only yields ensemble-averaged information on fluorophores passing through a relatively large $\left(\sim 0.5 \times 0.5 \times 1 \mu \mathrm{m}^{3}\right)$ open volume element. Alternatively, SPT can be used to detect the motion of small RNA molecules in a technique reported by Pitchiaya et al. (2012) termed intracellular single-molecule, highresolution localization, and counting (iSHiRLoC). Based on their molecular weights, microinjected "naked" doublestranded miRNAs and those loaded into "minimal" RISC complexes are expected to diffuse rapidly $\left(>10 \mu \mathrm{m}^{2} / \mathrm{s}\right.$ and $\sim 5 \mu \mathrm{m}^{2} / \mathrm{s}$, respectively [Ohrt et al. 2006]), blurring them out at the standard $100 \mathrm{~ms}$ exposure time required for sufficient fluorescent signal for SPT. Using microinjection and the HILO illumination strategy, Pitchiaya et al. (2012) reported that miRNAs chemically labeled on the $3^{\prime}$ end of the guide strand assembled into particles that could be tracked $1 \mathrm{~h}$ after microinjection into the cytosol. The resulting singleparticle trajectories were quantified to yield the diffusion rates of miRNAs at a given time point after microinjection. The study revealed two slowly diffusing populations that were assigned as mRNA-bound RISC RNPs, either diffusing freely (at $\sim 0.3 \mu \mathrm{m}^{2} / \mathrm{s}$ ) or docking (at $\sim 0.03 \mu \mathrm{m}^{2} / \mathrm{s}$ ) onto processing bodies (Pitchiaya et al. 2012).

A second technique within the iSHiRLoC toolbox is particle counting in fixed cells. Formaldehyde fixation captures the instantaneous position of all molecules in the cell. The rapid and stochastic photobleaching of single Cy5 dye molecules can then be exploited to count the number of fluorophores in individual bright spots, and this count, in turn, can be used as a proxy for the number of labeled molecules (Fig. 6D). Counting singly fluorophore-labeled miRNAs in this manner revealed that miRNA-containing complexes assembled significantly into multimers only in the presence of mRNA targets. Using two-color colocalization analysis, a fraction of these multimers were found associated with FP-tagged P-bodies, supporting the notion that miRNAs are recruited to P-bodies.

RNA silencing involves miRNA trafficking, strand unwinding, RISC-loading, target binding, and eventual degradation (Ha and Kim 2014). To examine all of these steps by visualizing them in live cells, Pitchiaya et al. (2017) included a third metric in iSHiRLoC, correlating the number of trajectories of mature miRISC.mRNPs diffusing in living cells with the total number of miRNA in fixed cells. Correlating the counts of live- and fixed-cell miRISC showed that miRNAs that efficiently load into RISC and engage target are present in comparable numbers in live and fixed cells, whereas miRNAs that stay in minimal complexes or remain "naked" (not loaded into the RISC complex) are only visible in fixed cells and instead blur out by virtue of the $100 \mathrm{~ms}$ exposure time used in live cell imaging (Fig. 6E). Correlating the counts of miRNAs, in the presence and absence of mRNA targets, allowed the investigators to build a detailed kinetic model of miRNA activity and turnover, which revealed that miRNAs not engaging a target mRNA are rapidly degraded (Pitchiaya et al. 2017).

These intracellular single-molecule visualization methods are not without limitations. The fluorescent dyes currently available for labeling have finite photostability under the illumination conditions required for single-molecule sensitivity, limiting the observation window of imaging. SPT is restricted by temporal resolution of imaging and is unsuitable for the study of very rapid molecular motion. Finally, microinjection currently has a low throughput of cells that can be targeted in each experiment, limiting the amount of data obtained from each specimen.

\section{CONCLUSIONS AND OUTLOOK}

As outlined in this survey, in vitro single-molecule fluorescence imaging of RNPs enables detailed characterization of the kinetics of RNA-protein interactions and accompanying conformational rearrangements. In vitro assays allow for specific manipulations of the reaction conditions and composition, such as specific placement of fluorophores and addition of cofactors, facilitating quantitative biophysical measurements. In principle, any RNP that can be prepared using genetic, chemical, and biochemical approaches can thus be studied at the single-molecule level. As more pathways of RNA metabolism are enumerated, the unique insights offered by SMFM tools will undoubtedly motivate their widespread adoption. The future will also likely see the proliferation of observables as additional labeling colors and parameters such as force measurements become more widely adopted. In vitro SMFM tools are poised to be further strengthened with other in vitro tools, such as high-resolution electron microscopy, that will enable direct correlation and integration of results from different singlemolecule observation modalities.

In parallel, complementary intracellular single-molecule tools such as iSHiRLoC have begun to reveal biological cornerstones of complex, multistep, and multicomponent RNA pathways such as RNA silencing. The key observables of intracellular tools such as the fluorescence intensities and diffusion trajectories of exogenous RNPs yield insights into cellular biology at defined probe concentrations, thereby overcoming the challenges of overexpression. The labeled components in these studies serve as molecular "baits" to 
A.P. Jalihal et al.

probe the complexity of the cellular milieu, which potentially differs considerably from classical in vitro assay conditions. The intracellular single-molecule toolbox thus paves the way toward a holistic study of RNP molecular biology by integrating biochemical analysis with insights into cell biology.

iSHiRLoC and other intracellular single-molecule fluorescence strategies face several challenges that need to be addressed. It is only a matter of time, however, until the current limitations will be overcome by, among others, improvements in fluorophores, delivery strategies, automation, microscopes, and analysis methods. Such advances will then permit a broader repertoire of observables that are currently only available to in vitro studies, such as measurements of intraparticle distances by smFRET and binding and dissociation rate constants by coincidence analysis. The future is thus bright for studying RNA and protein assemblies under the single-molecule fluorescence microscope both in vitro and in vivo for a full understanding of vast, still-emerging RNA worlds.

\section{ACKNOWLEDGMENTS}

The authors thank Julia Widom for a critical reading of this manuscript and acknowledge funding by National Institutes of Health Grants R01 GM062357, R01 GM118524, R01 GM122803, and R21 CA204560, and University of Michigan Comprehensive Cancer Center/Biointerfaces Institute and M-TRAC pilot grants. A.P.J. is supported by Cellular and Molecular Biology Training Grant T32GM007315.

\section{REFERENCES}

Abelson J, Blanco M, Ditzler MA, Fuller F, Aravamudhan P, Wood M, Villa T, Ryan DE, Pleiss JA, Maeder C, et al. 2010. Conformational dynamics of single pre-mRNA molecules during in vitro splicing. Nat Struct Mol Biol 17: 504-512.

Adumeau P, Sharma SK, Brent C, Zeglis BM. 2016. Site-specifically labeled immunoconjugates for molecular imaging-Part 2: Peptide tags and unnatural amino acids. Mol Imaging Biol 18: 153-165.

Agirrezabala X, Valle M. 2015. Structural insights into tRNA dynamics on the ribosome. Int J Mol Sci 16: 9866-9895.

Aitken CE, Marshall RA, Pulglisi JD. 2008. An oxygen scavenging system for improvement of dye stability in single-molecule fluorescence experiments. Biophys J 94: 1826-1835.

Al-Hashimi HM, Walter NG. 2008. RNA dynamics: It is about time. Curr Opin Struct Biol 18: 321-329.

Bacia K, Haustein E, Schwille P. 2014. Fluorescence correlation spectroscopy: Principles and applications. Cold Spring Harb Protoc 2014: 709725.

Blanco M, Walter NG. 2010. Analysis of complex single-molecule FRET time trajectories. Methods Enzymol 472: 153-178.

Blanco MR, Martin JS, Kahlscheuer ML, Krishnan R, Abelson J, Laederach A, Walter NG. 2015. Single molecule cluster analysis dissects splicing pathway conformational dynamics. Nat Meth 12: 1077-1084.
Buxbaum AR, Haimovich G, Singer RH. 2015. In the right place at the right time: Visualizing and understanding mRNA localization. Nat Rev Mol Cell Biol 16: 95-109.

Calloway NT, Choob M, Sanz A, Sheetz MP, Miller LW, Cornish VW. 2007. Optimized fluorescent trimethoprim derivatives for in vivo protein labeling. Chembiochem 8: 767-774.

Carrocci TJ, Lohe L, Ashton MJ, Hobartner C, Hoskins AA. 2017. Debranchase-resistant labeling of RNA using the 10DM24 deoxyribozyme and fluorescent modified nucleotides. Chem Commun 53: 11992-11995.

Chenouard N, Smal I, de Chaumont F, Maska M, Sbalzarini IF, Gong Y, Cardinale J, Carthel C, Coraluppi S, Winter M, et al. 2014. Objective comparison of particle tracking methods. Nat Meth 11: 281-289.

Chiu YL, Ali A, Chu CY, Cao H, Rana TM. 2004. Visualizing a correlation between siRNA localization, cellular uptake, and RNAi in living cells. Chem Biol 11: 1165-1175.

Cisse II, Kim H, Ha T. 2012. A rule of seven in Watson-Crick base-pairing of mismatched sequences. Nat Struct Mol Biol 19: 623-627.

Colomb W, Sarkar SK. 2015. Extracting physics of life at the molecular level: A review of single-molecule data analyses. Phys Life Rev 13: 107137.

Crawford DJ, Hoskins AA, Friedman LJ, Gelles J, Moore MJ. 2008. Visualizing the splicing of single pre-mRNA molecules in whole cell extract. RNA 14: 170-179.

Crawford DJ, Hoskins AA, Friedman LJ, Gelles J, Moore MJ. 2013. Singlemolecule colocalization FRET evidence that spliceosome activation precedes stable approach of $5^{\prime}$ splice site and branch site. Proc Natl Acad Sci 110: 6783-6788.

Crick F. 1970. Central dogma of molecular biology. Nature 227: 561-563.

Custer TC, Walter NG. 2017. In vitro labeling strategies for in cellulo fluorescence microscopy of single ribonucleoprotein machines. Protein Sci 26: 1363-1379.

Darre L, Ivani I, Dans PD, Gomez H, Hospital A, Orozco M. 2016. Small details matter: The $2^{\prime}$-hydroxyl as a conformational switch in RNA. J Am Chem Soc 138: 16355-16363.

DeHaven AC, Norden IS, Hoskins AA. 2016. Lights, camera, action! Capturing the spliceosome and pre-mRNA splicing with single-molecule fluorescence microscopy. WIREs RNA 7: 683-701.

dos Remedios CG, Moens PD. 1995. Fluorescence resonance energy transfer spectroscopy is a reliable "ruler" for measuring structural changes in proteins: Dispelling the problem of the unknown orientation factor. J Struct Biol 115: 175-185.

Edwards TE, Sigurdsson ST. 2014. Modified RNAs as tools in RNA biochemistry. In Handbook of RNA biochemistry (ed. Hartmann RK, Bindereif A, Schon A, Westhof E), pp. 36-52. Wiley, Weinheim, Germany.

Fauster K, Hartl M, Santner T, Aigner M, Kreutz C, Bister K, Ennifar E, Micura R. 2012. 2'-Azido RNA, a versatile tool for chemical biology: Synthesis, X-ray structure, siRNA applications, click labeling. ACS Chem Biol 7: 581-589.

Fei J, Wang J, Sternberg SH, MacDougall DD, Elvekrog MM, Pulukkunat DK, Englander MT, Gonzalez RL Jr. 2010. A highly purified, fluorescently labeled in vitro translation system for single-molecule studies of protein synthesis. Methods Enzymol 472: 221-259.

Gautier A, Juillerat A, Heinis C, Corrêa IR Jr, Kindermann M, Beaufils F, Johnsson K. 2008. An engineered protein tag for multiprotein labeling in living cells. Chem Biol 15: 128-136.

Gonzalez Bardeci N, Angiolini JF, De Rossi MC, Bruno L, Levi V. 2017. Dynamics of intracellular processes in live-cell systems unveiled by fluorescence correlation microscopy. IUBMB Life 69: 8-15.

Grima R, Walter NG, Schnell S. 2014. Single-molecule enzymology à la Michaelis-Menten. FEBS J 281: 518-530.

Ha M, Kim VN. 2014. Regulation of microRNA biogenesis. Nat Rev Mol Cell Biol 15: 509-524.

Hendrix C, Devreese B, Rozenski J, van Aerschot A, De Bruyn A, Van Beeumen J, Herdewijn P. 1995. Incorporation of 2 '-amido-nucleosides 
in oligodeoxynucleotides and oligoribonucleotides as a model for 2'-linked conjugates. Nucleic Acids Res 23: 51-57.

Hermanson GT. 2013. Nucleic acid and oligonucleotide modification and conjugation. In Bioconjugate techniques, 3rd ed. (ed. Hermanson GT), pp. 959-988. Academic, Boston.

Hickerson R, Majumdar ZK, Baucom A, Clegg RM, Noller HF. 2005. Measurement of internal movements within the $30 \mathrm{~S}$ ribosomal subunit using Förster resonance energy transfer. J Mol Biol 354: 459-472.

Hirsch M, Helm M. 2015. Live cell imaging of duplex siRNA intracellular trafficking. Nucleic Acids Res 43: 4650-4660.

Hocine S, Raymond P, Zenklusen D, Chao JA, Singer RH. 2013. Singlemolecule analysis of gene expression using two-color RNA labeling in live yeast. Nat Meth 10: 119-121.

Huang F, He J, Zhang Y, Guo Y. 2008. Synthesis of biotin-AMP conjugate for $5^{\prime}$ biotin labeling of RNA through one-step in vitro transcription. Nat Protoc 3: 1848-1861.

Hwang H, Myong S. 2014. Protein induced fluorescence enhancement (PIFE) for probing protein-nucleic acid interactions. Chem Soc Rev 43: 1221-1229.

Hwang W, Arluison V, Hohng S. 2011. Dynamic competition of DsrA and $r p o S$ fragments for the proximal binding site of Hfq as a means for efficient annealing. Nucleic Acids Res 39: 5131-5139.

Jens M, Rajewsky N. 2015. Competition between target sites of regulators shapes post-transcriptional gene regulation. Nat Rev Genet 16: 113126.

Johnson-Buck A, Su X, Giraldez MD, Zhao M, Tewari M, Walter NG. 2015. Kinetic fingerprinting to identify and count single nucleic acids. Nat Biotechnol 33: 730-732.

Juette MF, Terry DS, Wasserman MR, Zhou Z, Altman RB, Zheng Q, Blanchard SC. 2014. The bright future of single-molecule fluorescence imaging. Curr Opin Chem Biol 20: 103-111.

Kahlscheuer ML, Widom J, Walter NG. 2015. Single-molecule pull-down FRET to dissect the mechanisms of biomolecular machines. Methods Enzymol 558: 539-570.

Kim H, Abeysirigunawarden SC, Chen K, Mayerle M, Ragunathan K, Luthey-Schulten Z, Ha T, Woodson SA. 2014. Protein-guided RNA dynamics during early ribosome assembly. Nature 506: 334-338.

Kinoshita Y, Nishigaki K, Husimi Y. 1997. Fluorescence-, isotope- or biotin-labeling of the $5^{\prime}$-end of single-stranded DNA/RNA using T4 RNA ligase. Nucleic Acids Res 25: 3747-3748.

Knight SC, Xie L, Deng W, Guglielmi B, Witkowsky LB, Bosanac L, Zhang ET, El Beheiry M, Masson JB, Dahan M, et al. 2015. Dynamics of CRISPR-Cas9 genome interrogation in living cells. Science 350: 823826.

Kolb HC, Finn MG, Sharpless KB. 2001. Click chemistry: Diverse chemical function from a few good reactions. Angew Chem Int Ed Engl 40: 2004-2021.

Krishnan R, Blanco MR, Kahlscheuer ML, Abelson J, Guthrie C, Walter NG. 2013. Biased Brownian ratcheting leads to pre-mRNA remodeling and capture prior to first-step splicing. Nat Struct Mol Biol 20: 14501457.

Lakowicz JR. 2006. Principles of fluorescence spectroscopy. Springer, New York.

Larson JD, Rodgers ML, Hoskins AA. 2014. Visualizing cellular machines with colocalization single molecule microscopy. Chem Soc Rev 43: 1189-1200.

Lee TC, Kang M, Kim CH, Schultz PG, Chapman E, Deniz AA. 2016. Dual unnatural amino acid incorporation and click-chemistry labeling to enable single-molecule FRET studies of p97 folding. Chembiochem 17: 981-984.

Leontis NB, Stombaugh J, Westhof E. 2002. The non-Watson-Crick base pairs and their associated isostericity matrices. Nucleic Acids Res 30: 3497-3531.

Lerner E, Ploetz E, Hohlbein J, Cordes T, Weiss S. 2016. A quantitative theoretical framework for protein-induced fluorescence enhancementFörster-type resonance energy transfer (PIFE-FRET). J Phys Chem B 120: 6401-6410.
Levene MJ, Korlach J, Turner SW, Foquet M, Craighead HG, Webb WW 2003. Zero-mode waveguides for single-molecule analysis at high concentrations. Science 299: 682-686.

Li F, Dong J, Hu X, Gong W, Li J, Shen J, Tian H, Wang J. 2015. A covalent approach for site-specific RNA labeling in mammalian cells. Angew Chem Int Ed Engl 54: 4597-4602.

Liu Y, Lilley DMJ. 2017. Crystal structures of cyanine fluorophores stacked onto the end of double-stranded RNA. Biophys J 113: 23362343.

Liu CC, Schultz PG. 2010. Adding new chemistries to the genetic code. Annu Rev Biochem 79: 413-444.

Liu Y, Park J, Dahmen KA, Chemla YR, Ha T. 2010. A comparative study of multivariate and univariate hidden Markov modelings in timebinned single-molecule FRET data analysis. $J$ Phys Chem B 114: 5386-5403.

Liu Z, Lavis LD, Betzig E. 2015. Imaging live-cell dynamics and structure at the single-molecule level. Mol Cell 58: 644-659.

Los GV, Encell LP, McDougall MG, Hartzell DD, Karassina N, Zimprich C, Wood MG, Learish R, Ohana RF, Urh M, et al. 2008. HaloTag: A novel protein labeling technology for cell imaging and protein analysis. ACS Chem Biol 3: 373-382.

Lu J, Tsourkas A. 2009. Imaging individual microRNAs in single mammalian cells in situ. Nucleic Acids Res 27: e100.

Lu HP, Xun L, Xie XS. 1998. Single-molecule enzymatic dynamics. Science 282: 1877-1882.

Lyon K, Stasevich TJ. 2017. Imaging translational and post-translational gene regulatory dynamics in living cells with antibody-based probes. Trends Genet 33: 322-335.

Mamot A, Sikorski PJ, Warminski M, Kowalska J, Jemielity J. 2017. Azido-functionalized $5^{\prime}$ cap analogues for the preparation of translationally active mRNAs suitable for fluorescent labeling in living cells. Angew Chem Int Ed Engl 56: 15628-15632.

McCann JJ, Choi UB, Zheng L, Weninger K, Bowen ME. 2010. Optimizing methods to recover absolute FRET efficiency from immobilized single molecules. Biophys J 99: 961-970.

McNally JG. 2008. Quantitative FRAP in analysis of molecular binding dynamics in vivo. Methods Cell Biol 85: 329-351.

Michelini F, Pitchiaya S, Vitelli V, Sharma S, Gioia U, Pessina F, Cabrini M, Wang Y, Capozzo I, Iannelli F, et al. 2017. Damage-induced lncRNAs control the DNA damage response through interaction with DDRNAs at individual double-strand breaks. Nat Cell Biol 19: $1400-1411$

Milas P, Gamari BD, Parrot L, Krueger BP, Rahmanseresht S, Moore J, Goldner LS. 2013. Indocyanine dyes approach free rotation at the $3^{\prime}$ terminus of A-RNA: A comparison with the $5^{\prime}$ terminus and consequences for fluorescence resonance energy transfer. J Phys Chem B 117: 8649-8658.

Moffitt JR, Chemla YR, Bustamante C. 2010. Methods in statistical kinetics. Methods Enzymol 475: 221-257.

Monnier N, Barry Z, Park HY, Su KC, Katz Z, English BP, Dey A, Pan K, Cheeseman IM, Singer RH, et al. 2015. Inferring transient particle transport dynamics in live cells. Nat Meth 12: 838-840.

Morisaki T, Lyon K, DeLuca KF, DeLuca JG, English BP, Zhang Z, Lavis LD, Grimm JB, Viswanathan S, Looger LL, et al. 2016. Real-time quantification of single RNA translation dynamics in living cells. Science 352: $1425-1429$.

Mustoe AM, Brooks CL, Al-Hashimi HM. 2014. Hierarchy of RNA functional dynamics. Annu Rev Biochem 83: 441-466.

Nissen P, Ippolito JA, Ban N, Moore PB, Steitz TA. 2001. RNA tertiary interactions in the large ribosomal subunit: The A-minor motif. Proc Natl Acad Sci 98: 4899-4903.

Ohrt T, Merkle D, Birkenfeld K, Echeverri CJ, Schwille P. 2006. In situ fluorescence analysis demonstrates active siRNA exclusion from the nucleus by exportin 5. Nucleic Acids Res 34: 1369-1380.

Ohrt T, Mutze J, Staroske W, Weinmann L, Hock J, Crell K, Meister G, Schwille P. 2008. Fluorescence correlation spectroscopy and fluorescence cross-correlation spectroscopy reveal the cytoplasmic origina- 
A.P. Jalihal et al.

tion of loaded nuclear RISC in vivo in human cells. Nucleic Acids Res 36: 6439-6449.

Okumus B, Wilson TJ, Lilley DM, Ha T. 2004. Vesicle encapsulation studies reveal that single molecule ribozyme heterogeneities are intrinsic. Biophys J 87: 2798-2806.

Pan C, Potratz JP, Cannon B, Simpson ZB, Ziehr JL, Tijerina P, Russell R. 2014. DEAD-box helicase proteins disrupt RNA tertiary structure through helix capture. PLoS Biol 12: e1001981.

Paredes E, Evans M, Das SR. 2011. RNA labeling, conjugation and ligation. Methods 54: 251-259.

Pitchiaya S, Androsavich JR, Walter NG. 2012. Intracellular single molecule microscopy reveals two kinetically distinct pathways for microRNA assembly. EMBO Rep 13: 709-715.

Pitchiaya S, Krishnan V, Custer TC, Walter NG. 2013. Dissecting noncoding RNA mechanisms in cellulo by single-molecule high-resolution localization and counting. Methods 63: 188-199.

Pitchiaya S, Heinicke LA, Park JI, Cameron EL, Walter NG. 2017. Resolving subcellular miRNA trafficking and turnover at single-molecule resolution. Cell Rep 19: 630-642.

Rasnik I, McKinney SA, Ha T. 2006. Nonblinking and long-lasting singlemolecule fluorescence imaging. Nat Meth 3: 891-893.

Rinaldi AJ, Suddala KC, Walter NG. 2015. Native purification and labeling of RNA for single molecule fluorescence studies. Methods Mol Biol 1240: 63-95.

Rinaldi AJ, Lund PE, Blanco MR, Walter NG. 2016. The Shine-Dalgarno sequence of riboswitch-regulated single mRNAs shows ligand-dependent accessibility bursts. Nat Commun 7: 8976.

Ritter JG, Veith R, Veenendaal A, Siebrasse JP, Kubitscheck U. 2010. Light sheet microscopy for single molecule tracking in living tissue. PLoS ONE 5: e11639.

Roy R, Hohng S, Ha T. 2008. A practical guide to single-molecule FRET. Nat Meth 5: 507-516.

Salomon WE, Jolly SM, Moore MJ, Zamore PD, Serebrov V. 2015. Singlemolecule imaging reveals that Argonaute reshapes the binding properties of its nucleic acid guides. Cell 162: 84-95.

Schmidt JC, Zaug AJ, Cech TR. 2016. Live cell imaging reveals the dynamics of telomerase recruitment to telomeres. Cell 166: 11881197.

Schulz D, Rentmeister A. 2014. Current approaches for RNA labeling in vitro and in cells based on click reactions. Chembiochem 15: 23422347.

Semlow DR, Blanco MR, Walter NG, Staley JP. 2016. Spliceosomal DEAH-box ATPases remodel pre-mRNA to activate alternative splice sites. Cell 164: 985-998.

Senavirathne G, Liu J, Lopez MA Jr, Hanne J, Martin-Lopez J, Lee JB, Yoder KE, Fishel R. 2015. Widespread nuclease contamination in commonly used oxygen-scavenging systems. Nat Meth 12: 901-902.

Shcherbakova I, Hoskins AA, Friedman LJ, Serebrov V, Corrêa IR Jr, Xu MQ, Gelles J, Moore MJ. 2013. Alternative spliceosome assembly pathways revealed by single-molecule fluorescence microscopy. Cell Rep 5: 151-165.

Shen K, Arslan S, Akopian D, Ha T, Shan So. 2012. Activated GTPase movement on an RNA scaffold drives co-translational protein targeting. Nature 492: 271-275.

Shen H, Tauzin LJ, Baiyasi R, Wang W, Moringo N, Shuang B, Landes CF. 2017. Single particle tracking: From theory to biophysical applications. Chem Rev 117: 7331-7376.
Shi J, Dertouzos J, Gafni A, Steel D. 2008. Application of single-molecule spectroscopy in studying enzyme kinetics and mechanism. Methods Enzymol 450: 129-157.

Solomatin S, Herschlag D. 2009. Methods of site-specific labeling of RNA with fluorescent dyes. Methods Enzymol 469: 47-68.

Stewart MP, Sharei A, Ding X, Sahay G, Langer R, Jensen KF. 2016. In vitro and ex vivo strategies for intracellular delivery. Nature 538: 183192.

Sun X, Zhang A, Baker B, Sun L, Howard A, Buswell J, Maurel D, Masharina A, Johnsson K, Noren CJ, et al. 2011. Development of SNAPtag fluorogenic probes for wash-free fluorescence imaging. Chembiochem 12: 2217-2226.

Swoboda M, Henig J, Cheng HM, Brugger D, Haltrich D, Plumere N, Schlierf M. 2012. Enzymatic oxygen scavenging for photostability without pH drop in single-molecule experiments. ACS Nano 6: 6364-6369.

Thorn K. 2017. Genetically encoded fluorescent tags. Mol Biol Cell 28: 848-857.

Tirat A, Freuler F, Stettler T, Mayr LM, Leder L. 2006. Evaluation of two novel tag-based labelling technologies for site-specific modification of proteins. Int J Biol Macromol 39: 66-76.

Tokunaga M, Imamoto N, Sakata-Sogawa K. 2008. Highly inclined thin illumination enables clear single-molecule imaging in cells. Nat Meth 5: $159-161$.

Toomre D. 2012. Cellular imaging using total internal reflection fluorescence microscopy: Theory and instrumentation. Cold Spring Harb Protoc 2012: 414-424.

Trachman RJ 3rd, Truong L, Ferre-D'Amare AR. 2017. Structural principles of fluorescent RNA aptamers. Trends Pharmacol Sci 38: 928-939.

Tsai A, Puglisi JD, Uemura S. 2016. Probing the translation dynamics of ribosomes using zero-mode waveguides. Prog Mol Biol Transl Sci 139: $1-43$.

Turunen JJ, Pavlova LV, Hengesbach M, Helm M, Müller S, Hartmann RK, Frilander MJ. 2014. RNA ligation. In Handbook of RNA biochemistry (ed. Hartmann RK, Bindereif A, Schön A, Westhof E), pp. 45-88. Wiley, Weinheim, Germany.

Tyagi S. 2009. Imaging intracellular RNA distribution and dynamics in living cells. Nat Meth 6: 331-338.

Wahl MC, Will CL, Lührmann R. 2009. The spliceosome: Design principles of a dynamic RNP machine. Cell 136: 701-718.

Walter NG, Huang CY, Manzo AJ, Sobhy MA. 2008. Do-it-yourself guide: How to use the modern single-molecule toolkit. Nat Meth 5: 475-489.

Wang J, Caban K, Gonzalez RL Jr. 2015. Ribosomal initiation complexdriven changes in the stability and dynamics of initiation factor 2 regulate the fidelity of translation initiation. J Mol Biol 427: 18191834.

Willkomm DK, Hartmann RK. 2014. 3'-Terminal attachment of fluorescent dyes and biotin. In Handbook of RNA biochemistry (ed. Hartmann RK, Bindereif A, Schön A, Westhof E), pp. 117-128. Wiley, Weinheim, Germany.

Wu RA, Dagdas YS, Yilmaz ST, Yildiz A, Collins K. 2015. Single-molecule imaging of telomerase reverse transcriptase in human telomerase holoenzyme and minimal RNP complexes. eLife 4: e08363.

Yan X, Hoek TA, Vale RD, Tanenbaum ME. 2016. Dynamics of translation of single mRNA molecules in vivo. Cell 165: 976-989.

Yasuda R, Masaike T, Adachi K, Noji H, Itoh H, Kinosita K Jr. 2003. The ATP-waiting conformation of rotating F1-ATPase revealed by singlepair fluorescence resonance energy transfer. Proc Natl Acad Sci 100: 9314-9318. 


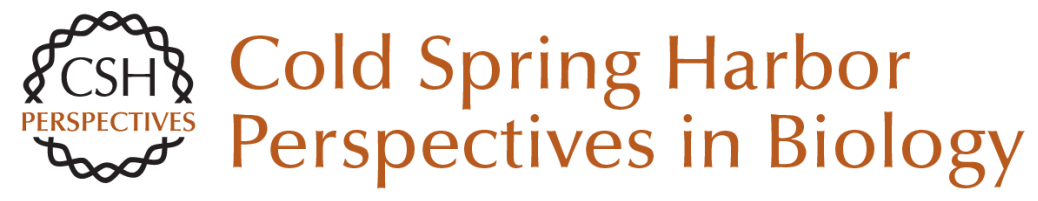

\section{Coming Together: RNAs and Proteins Assemble under the Single-Molecule Fluorescence Microscope}

Ameya P. Jalihal, Paul E. Lund and Nils G. Walter

Cold Spring Harb Perspect Biol 2019; doi: 10.1101/cshperspect.a032441

Subject Collection RNA Worlds

Alternate RNA Structures

Marie Teng-Pei Wu and Victoria D'Souza

Approaches for Understanding the Mechanisms of Long Noncoding RNA Regulation of Gene Expression

Patrick McDonel and Mitchell Guttman

Principles and Practices of Hybridization Capture

Experiments to Study Long Noncoding RNAs That

Act on Chromatin

Matthew D. Simon and Martin Machyna

Linking RNA Sequence, Structure, and Function

on Massively Parallel High-Throughput

Sequencers

Sarah K. Denny and William J. Greenleaf

Extensions, Extra Factors, and Extreme

Complexity: Ribosomal Structures Provide

Insights into Eukaryotic Translation

Melanie Weisser and Nenad Ban

Nascent RNA and the Coordination of Splicing with Transcription

Karla M. Neugebauer

Combining Mass Spectrometry (MS) and Nuclear Magnetic Resonance (NMR) Spectroscopy for Integrative Structural Biology of Protein-RNA

Complexes

Alexander Leitner, Georg Dorn and Frédéric H.-T. Allain

For additional articles in this collection, see http://cshperspectives.cshlp.org/cgi/collection/

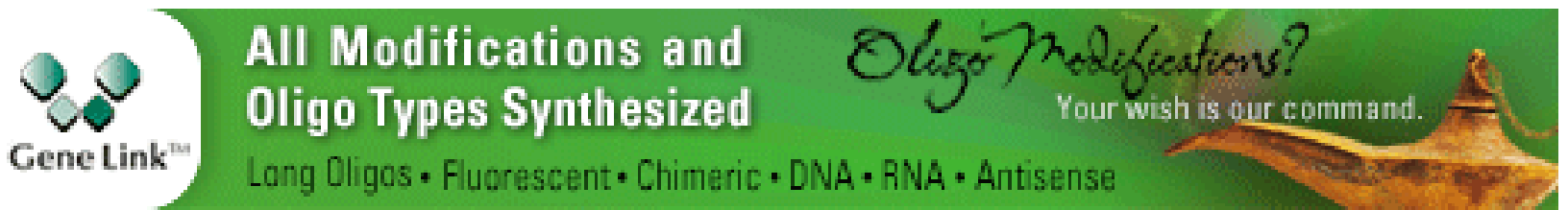

Structural Biology of Telomerase

Yaqiang Wang, Lukas Susac and Juli Feigon

Structural Insights into Nuclear pre-mRNA

Splicing in Higher Eukaryotes

Berthold Kastner, Cindy L. Will, Holger Stark, et al.

What Are 3' UTRs Doing?

Christine Mayr

Single-Molecule Analysis of Reverse

Transcriptase Enzymes

Linnea I. Jansson and Michael D. Stone

CRISPR Tools for Systematic Studies of RNA

Regulation

Jesse Engreitz, Omar Abudayyeh, Jonathan

Gootenberg, et al.

Relating Structure and Dynamics in RNA Biology Kevin P. Larsen, Junhong Choi, Arjun Prabhakar, et al.

Beyond DNA and RNA: The Expanding Toolbox of Synthetic Genetics

Alexander I. Taylor, Gillian Houlihan and Philipp Holliger

Copyright (C) 2019 Cold Spring Harbor Laboratory Press; all rights reserved 
Discovering and Mapping the Modified Nucleotides That Comprise the Epitranscriptome of mRNA

Bastian Linder and Samie R. Jaffrey
Structural Basis of Nuclear pre-mRNA Splicing:

\section{Lessons from Yeast}

Clemens Plaschka, Andrew J. Newman and Kiyoshi Nagai

For additional articles in this collection, see http://cshperspectives.cshlp.org/cgi/collection/

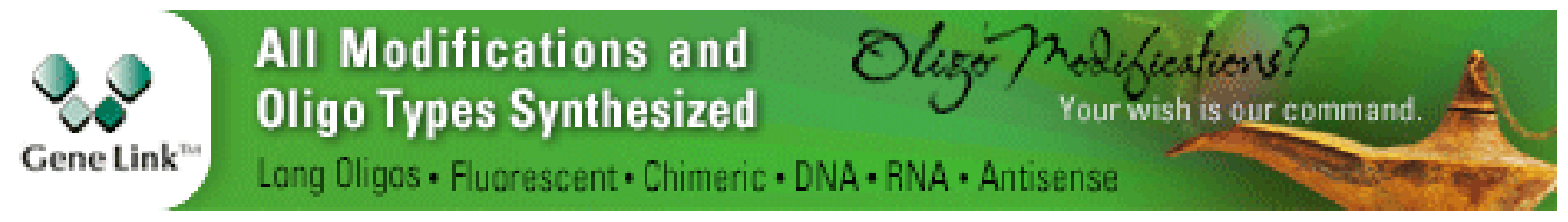

Copyright (C) 2019 Cold Spring Harbor Laboratory Press; all rights reserved 